\title{
Surface Patterning Increases Fluid Sorption Efficiency in Porous Reactive Coatings: A Model for Optimised Surface-Flow Filtration
}

\author{
P. A. C. Gane ${ }^{1,3} \cdot$ C. J. Ridgway ${ }^{2} \cdot$ M. Kijevčanin ${ }^{3} \cdot$ M. Stijepović ${ }^{3} \cdot$ P. S. Uskoković ${ }^{3}$. \\ N. Barać ${ }^{4} \cdot$ K. Dimić-Mišić ${ }^{1} \cdot$ M. Imani ${ }^{1} \cdot$ D. Janaćkovićc ${ }^{3} \cdot$ E. Barceló ${ }^{1,2}$
}

Received: 13 July 2020 / Accepted: 26 May 2021 / Published online: 3 June 2021

(C) The Author(s) 2021

\begin{abstract}
A novel surface-flow filter has been designed to take advantage of a combination of both diffusion and lateral flow permeation, since active coatings, even if porous, tend to be far less permeable than through-flow constructs. The formation of a continuous coating layer virtually excludes any fluid transport into the coating other than by planar diffusion for gases or capillarity for liquids. This study considers the additional potential advantage of creating a pixelated/patterned coating, applied using a pin coater to form printed dots onto a highly permeable cellulose fibrous paper-like substrate. The coating-fine particulate calcium carbonate combined with micro-nanofibrillated cellulose as binder and humectant-reacts on exposure to $\mathrm{NO}_{2}$ gas to form calcium nitrate. Experimental results show an effective doubling of nitrate-forming efficiency using pixelated coating compared with a reference continuous layer coating. To establish an understanding of the comparative mechanisms of gas-coating contact, computational fluid dynamic modelling is used to generate surface pressure profiles combined with a pore network model of the coating to estimate theoretical fluid permeability and gas diffusion coefficients. Although pressure-driven permeation was calculated to be approximately two orders of magnitude less than the diffusive flow, it is concluded that patterned aerofoil pressure differential effects can reduce the impact of surface stagnant layering and so aid fluid transfer, boosting the diffusive transport, which in turn delivers greater contact efficiency based on the increased accessibility to the active coating.
\end{abstract}

P. A. C. Gane

patrick.gane@aalto.fi

1 School of Chemical Engineering, Department of Bioproducts and Biosystems, Aalto University, 00076 Aalto, Helsinki, Finland

2 Omya International AG, Baslerstrasse 42, 4665 Oftringen, Switzerland

3 Faculty of Technology and Metallurgy, University of Belgrade, Karnegijeva 4, 11200 Belgrade, Serbia

4 Innovation Center of Faculty of Technology and Metallurgy Ltd., Karnegijeva 4, 11200 Belgrade, Serbia 


\section{Article Highlights}

- Effective doubling of surface-flow filter gas sorption achieved by patterning/pixelation of microporous reactive coating.

- Surface flow and pore network modelling show that coating permeability is enhanced by porous particle inclusion and pressure gradients induced by Bernoulli effect.

- Calculated average diffusion coefficient shown to be approximately two orders of magnitude greater than pressure-driven permeation.

Keywords $\mathrm{NO}_{x}$-mitigation $\cdot$ Surface-flow reactive filtration $\cdot$ Pore network and computational fluid dynamic modelling · Gas/liquid permeation and diffusion · Transport in porous media

\section{Introduction}

Surface-flow filters have two main advantages versus through-flow filtration, namely reduced energy consumption since the bulk flow does not pass through a fine pore matrix, and particulate blocking is largely avoided, Fig. 1. However, species reactive surfaces in surface-flow systems suffer from reduced fluid volume-to-contact area ratio, not only due to the contact being via a single exposed surface but also due to the formation of a surface stagnant layer, which inhibits freshly replenished contact with the reactive filter material.

Many examples of high energy consumption through-flow filtration, and therefore at high environmental cost, exist. Examples of such include recycled drinking water filtration using activated carbon blocks to remove organic contaminants including endocrine modifying substances and pharmaceutical active ingredients, air filtration in buildings in heavily polluted city areas, and in vehicle cabin filters to remove both particulate and gaseous pollutants, to name but a few. In all of these cases, both maintenance of the filters and low selective removal efficiency towards the end of the cartridge/filter element lifetime add a high cost burden, resulting in improper use under inefficient conditions over extended periods. Therefore, if the ability to use efficient surface-flow filters, including electrostatic, plasma, or surface reactive elements, were to be increased, the environmental benefit could be extensive.

Of major interest, especially in city environments, and increasingly inside vehicles or industrial plant, is the drive to reduce human exposure to oxides of nitrogen $\left(\mathrm{NO}_{x}\right)$. Wherever combustion in air at high temperature and pressure occurs, even for carbon-free combustibles such as hydrogen, $\mathrm{NO}_{x}$ is produced as the direct result of oxidation of the major component in air, nitrogen. The economic and humanitarian health benefits of removing $\mathrm{NO}_{x}$, a pulmonary irritant, from breathed air are recognised universally throughout regulatory and governmental agencies. Although carbon fuel-based motorised transport has been the main focus for the environmental lobby, the impact of, for example, the comparative planetary rarity of lithium and cobalt for battery production and the resultant recycling challenges and waste generation after use is becoming increasingly discussed (Steward et al. 2019). Combustion, avoiding $\mathrm{CO}_{2}$ and other greenhouse gas emissions, therefore, remains an attractive option, but as a result $\mathrm{NO}_{x}$ will remain a problem to be dealt with. It is based on this premise that our previously reported work (Gane et al. 2020) on developing 
Fig. 1 Contamination of a through-flow filter can be greatly reduced using a b surface-flow concept. Further enhancement can be realised using an active surface-textured/patterned (pixelated) gas-sorbing coating
Through-flow

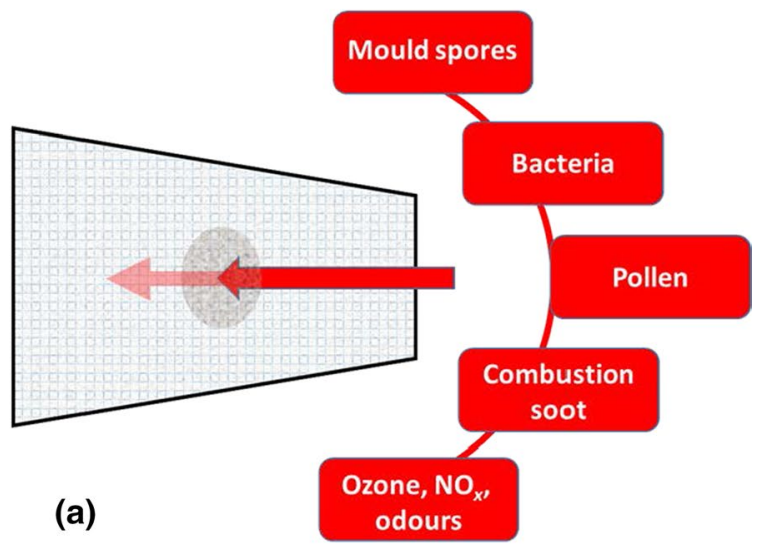

\section{Surface-flow}

patterned (pixelated) surface active coating

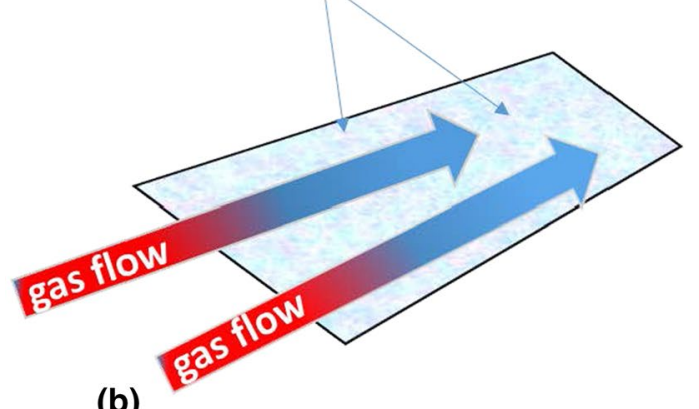

a surface-flow reactive filter for $\mathrm{NO}_{2}$ sorption included proposing a way to increase sorption performance of a planar coating applied to an essentially flat recycled paper surface. The principle underlying the capture of $\mathrm{NO}_{2}$ relies on reacting a porous permeable surface coating comprising ground limestone particles (calcium carbonate) and a bio-sourced cellulose humectant. Our practical solution was to consider firstly increasing the permeability of the porous structure, achieved by adding a spacer mineral, in the exemplified case a volcanic glass (expanded perlite), and secondly to devise a means of surface patterning the coating layer. This patterning, or pixelation, was achieved by using a needle/pin coater applying highly viscous droplets of aqueous coating suspension onto the substrate to form an hexagonal array of printed dots. Increased gas sorption was successfully observed by making these changes, but clearly the question remained open on what exactly the mechanism of patterning might be and how that mechanism could be improved further for the more generalised case.

Very little appears in the prior literature regarding surface-flow filtration, and in particular an absence of study of patterned coatings for filtration. What prior art there is concerning patterned surfaces frequently refers to the problem of fouling of sea vessel hulls, which does not address directly the problem considered here. Nonetheless, modelling in the case 
of fouling showed that turbulence can be generated around specially sized and spaced surface patterning, which acts to prevent stagnant layer formation (Myan et al. 2018). Of additional relevance to our case is the modelling of turbulence in air circulation in high-rise building-lined city streets (Vardoulakis et al. 2003), which approaches the issue of transporting pollution and the value of air circulation to achieve this, though not addressing filtration per se.

Furthermore, surface-flow sorption, as used in this work, differs significantly from the generally applied tangential flow mechanisms typically reported in the prior art, for example (Belfort et al. 1994; Busatto et al. 2018; van Reis et al. 1997). Tangential, often referred to as cross-flow, filtration essentially drives the passage of a limited size-selected species through a membrane by application of an external pressure as the carrier medium and other larger species flow tangentially across the membrane surface, where the external pressure difference is applied within a closed system. In the mirror image case of osmotic transportation of the medium, the pressure arises from the concentration difference of the carried species rather than size filtration without any external pressure being applied. On the contrary, in the case of reverse osmosis cross-flow filtration, a common example of which is water desalination, the osmotic pressure is opposed by an externally applied pressure so as to move water in the opposite direction from high salt saturation to low/zero salt content through a semipermeable membrane blocking the transport of the salt (Christy et al. 2002).

In this study of sorption by reaction with the substrate under tangential flow, the contact between the species being sorbed and the reactive substrate is enabled by the passage of the permeating total fluid mix, i.e. no species separation occurs prior to permeation through the porous network of the designed substrate. This defines the fundamental difference between the mechanisms under research in this work versus species size-selective pressure generation through external or pre-existing concentration forces. Thus, in the absence of externally applied pressure, flow of the total fluid mix can be enhanced either by the tangential flow dynamic itself, hence the need to design the surface accordingly to generate pressure gradients, as will be described, and/or by the depletion of a given species due to the reactive sorption of that species. It is, then, by the action of sorbing by progressive reaction that differential component concentration gradients within the porous substrate network are generated. To meet the challenge of $\mathrm{NO}_{2}$ capture, as described above, the sorption of $\mathrm{NO}_{2}$ is driven by reaction with calcium carbonate to form calcium nitrate salt. The depletion of $\mathrm{NO}_{2}$ in the carrier air mix by this process leads to a molecular sink supporting a diffusive concentration-driven selection for the transportation of $\mathrm{NO}_{2}$ to the reaction sites, and hence selective permeation of $\mathrm{NO}_{2}$ through the substrate pore network. We set out to compare the relative impact of these two mechanisms, namely surface-flow-induced permeation versus diffusive permeation.

The closest prior art comes in two forms, (1) that applying to long pathway diffusion without tangential flow, and (2) tangential flow over an ultrathin membrane. Case (1) applies to such considerations as permeation of gases through construction materials. The very practical example given by Vermesi et al. (2018) describes how the transmission of carbon monoxide through a gypsum wall panel is shown to be "surprisingly" fast, illustrating the dangers to inhabitants in a building from $\mathrm{CO}$ poisoning even though no direct ventilation connection is present. Such a situation represents diffusion through thick, microporous, structural materials with relatively long permeation pathways. Case (2) relates to comparing the capture and release of nanoparticles and extracellular vesicles on conventional and ultrathin membranes under tangential flow (Dehghani et al. 2019), in which nanoporous silicon nitride is used as the membrane to capture nanoparticles formed in a tangentially flowing plasma. However, despite the functional similarities, the ultrathin nature of the membrane, defined as having a 
thickness of the same order of magnitude as the pore size, renders the conclusions from their microfluidic modelling, assuming continuous perpendicular flow through the membrane in response to external pressure, of only qualitative value in our case. Nonetheless, some of the observations from both these cases provide a useful indicator of the system-limiting factors. For example, we can take advantage of the reported "surprisingly" fast diffusion of gas given a sufficiently high concentration gradient even over long path lengths, though the path length we choose is much shorter in a thin coating layer and so diffusion will be expected to be very rapid. This can be combined with the evidence in the second case that ultrathin membranes are ideally suited for tangential flow analyte capture for two reasons, firstly, the operating pressures are orders-of-magnitude lower for ultrathin membranes than for membranes with conventional thicknesses $(1-10 \mu \mathrm{m})$, and, secondly, particles that are captured are associated with a surface, rather than trapped in a bulk-matrix. We particularly wish to take advantage of these benefits using the coating layer as a thin filter, but placed on a thicker base substrate which is not participating in reaction/capture, although being highly permeable itself. Using this construct serves to isolate the permeation of interest to the top thin layer only, with enhanced access to the layer. In so doing, this allows us to develop a filter function that does not require high external energy to generate tangential flow, but rather taking advantage of diffusion of fluid (gas in our case) to provide the transport mechanism. Therefore, to differentiate between the mechanisms at play in our design from those of either static, or conventional, or ultrathin, tangential flow filtration, we define the method we adopt as 'surface-flow filtration'. This definition takes into account the functionality of the coating layer alone under tangential fluid flow, without external applied pressure and without the cross-flow properties of a bulk membrane, to act to renew the feed gas concentration, and, with the special pixelated coating, to generate localised pressure gradients.

Our study, therefore, moves the discussion forward by analysing the likelihood of pressure differences, arising from surface structuration, used to drive fluid permeation across surfaceflow filters. In the case of a gas, and the desire for it to be directed through a surface porous structure, we alternatively rely on the generation of pressure gradients together with diffusion, rather than turbulence. Here, to analyse and describe such flow phenomena, we undertake two-dimensional (2D) finite element fluid flow modelling, and combine it with experimental intrusion porosimetric and fluid permeability analyses of the coating pore structure. To realise a model of the coating pore network, the porosimetry intrusion curves of comparative coating structures are represented as a 3D Cartesian construct of cubic pores and cylindrical connecting throats, using the PoreXpert software suite (PoreXpert Ltd., Davy 601 Laboratories, Plymouth University, Plymouth, Devon, United Kingdom), from which the relative permeability of both liquid (Darcy flow) and air $\left(\mathrm{N}_{2}\right)$ can be scaled to the absolute value of the experimental fluid (Gribble et al. 2011). Thus, knowing the permeability, the permeation rate of gas under the pressure gradient of the surface flow around the printed coating dot can be estimated. This is then compared with the diffusion-only defined rate derived from the coating porosity and average diffusion coefficient, in ratio to that of $\mathrm{NO}_{2}$ gas in air, using the expression offered by Cape (2005) following the work of Berkowitz and Balberg (1993), Hunt (2001), and Hunt and Ewing $(2003,2009)$. The target can, therefore, be met to provide a generalised methodology for developing and optimising increased efficiency of surface-flow filtration. 
Fig. 2 Multiply recycled newsprint fibre: optical microscopy image taken using a Leica DM 750 (Leica Microsystems GmbH, Ernst-Leitz-Strasse 17-37, Wetzlar, 35578 Germany)_red scale bars indicate fibre dimensions superposed at the artificially coloured (yellow-green) image software sites, which indicate also residual ink pigment (dots): (modified from Gane et al. 2020: copyright held by same authors as this paper)

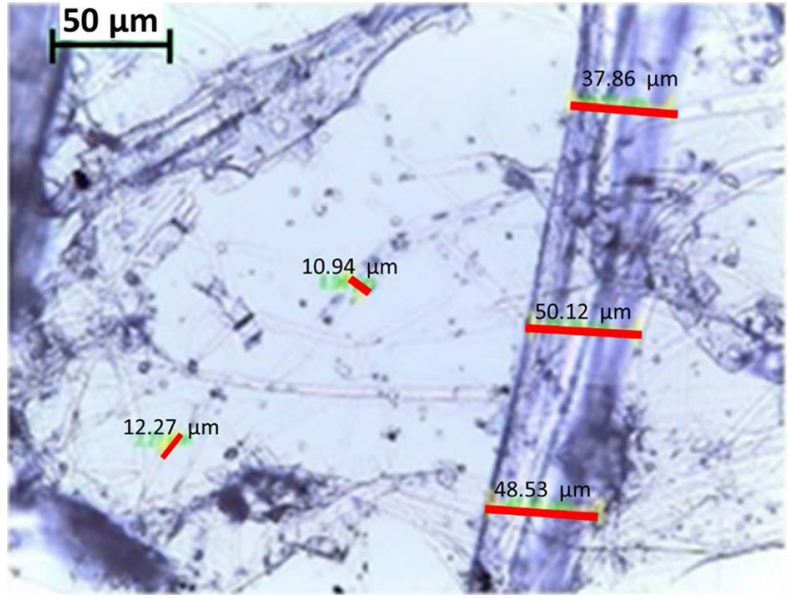

Table 1 Handsheet substrate properties made from multiply recycled newsprint

\begin{tabular}{|c|c|c|c|c|c|c|c|}
\hline & $\begin{array}{l}\text { Area } \\
\left(\mathrm{m}^{2}\right)\end{array}$ & $\begin{array}{l}\text { Mass } \\
\text { (g) }\end{array}$ & $\begin{array}{l}\text { Basis weight } \\
\left(\mathrm{g} \mathrm{m}^{-2}\right)\end{array}$ & $\begin{array}{l}\text { Thickness } \\
\text { (caliper) } \\
(\mu \mathrm{m})\end{array}$ & $\begin{array}{l}\text { Volume } \\
\left(\mathrm{cm}^{3}\right)\end{array}$ & $\begin{array}{l}\text { Density } \\
\left(\mathrm{g} \mathrm{cm}^{-3}\right)\end{array}$ & $\begin{array}{l}\text { Porosity } \\
\text { (\%) }\end{array}$ \\
\hline Recycled newsprint sheet & 0.027 & 2.18 & 80 & 183 & 4.98 & 0.44 & 70.9 \\
\hline
\end{tabular}

\section{Experimental}

\subsection{Materials and Methods}

\subsubsection{Filter Substrate}

The substrate for the active filter coating comprised recycled printed newsprint that was multiply re-pulped to simulate cellulose fibres that have been recycled over and over again until they are no longer suitable for standard paper or board manufacture, due to structural weakening and loss of hydrogen bonding potential, Fig. 2. The purpose of using such an over-recycled fibre source was primarily to meet the criteria of placing this $\mathrm{NO}_{x}$ filter design in the circular economy, and for this application, applying low coating forces, the strength properties of the substrate were found to suffice. Following disintegration, the aqueous fibre suspension was diluted to $1 \mathrm{w} / \mathrm{w} \%$ in a high shear laboratory mixer. The suspension was then formed into a handsheet by vacuum draining, pressing, and drying, as described in the previous work by Gane et al. (2020). Substrate properties are shown in Table 1.

\subsubsection{Active Coating}

We use the example of an active coating formulated to promote the reaction for calcium carbonate, in the form of ground limestone or marble (GCC), with $\mathrm{NO}_{2}$ gas in 
the presence of moisture, as shown in Eq. (1), such that calcium nitrate $\left(\mathrm{Ca}\left(\mathrm{NO}_{3}\right)_{2}\right)$ is generated. The value in circular economy is based on calcium nitrate being considered a premium fertiliser for use in horticulture as a concentrate or hydroponic nutrient, and, in slurry dilution, for agricultural crops and sustainable forestry plantations (Hignett, 1985).

$$
\mathrm{CaCO}_{3}+\mathrm{H}_{2} \mathrm{O}+2 \mathrm{NO}_{2}+1 / 2 \mathrm{O}_{2} \rightarrow \mathrm{Ca}\left(\mathrm{NO}_{3}\right)_{2}+\mathrm{H}_{2} \mathrm{CO}_{3}
$$

As we see from Eq. (1), the reaction between $\mathrm{NO}_{2}$ and $\mathrm{CaCO}_{3}$ is not one of simple adsorption but includes the need for a low level of moisture to be present. If high levels of moisture are present causing liquid condensation, then $\mathrm{Ca}\left(\mathrm{NO}_{2}\right)_{3}$ is undesirably formed due to dissolution of $\mathrm{NO}_{2}$ in water and the resulting formation of nitrous acid. Therefore, to ensure sufficient but controlled amounts of in-situ moisture, a humectant is used.

\subsubsection{Micro-Nanofibrillated Cellulose (MNFC) as Binder + Humectant}

Conveniently, finely nanofibrillated cellulose fibres act both as a binder and humectant, due to their affinity to water vapour (hygroscopy). Fibrillated cellulose was produced from the same fibre source as the substrate by passing the disintegrated fibre pulp suspension through a super masscolloider (Model MKZA10-15IV, Masuko Sangyo Co. Ltd., 1-12-24 Honcho, Kawaguchi-city, Saitama-pref, JAPAN), where the fibres are ground between a static and an abrasive stone rotating at a speed of $1500 \mathrm{~min}^{-1}(\mathrm{rpm})$ until a gel-like material suspension is formed indicating nanofibrillation has occurred (Li et al. 2014). The fibrillation occurs under the hydrodynamic pressure, mechanical grinding, and shearing forces generated by the grinding stones, resulting in the breakdown of the macroscopic plant fibre walls and the release of nanometre-thick strands of nanofibrils branching from the parent fibre wall. This combination of reduction to microfibrils with nanofibrils branching from the surface gives the material the nomenclature used here: micro-nanofibrillated cellulose (MNFC) (Dimić-Mišić et al. 2013a, b; Schenker et al. 2016), and shown in the electron microscope image in Fig. 3.

Fig. 3 Electron microscope image of MNFC produced from multiply recycled newsprint showing the micro- and nanofibrils together with retained filler and ink particles (image taken on a JEM-2200FS; JEOL Ltd., Akashima, Tokyo, Japan)—red scale bars indicate microfibril and residual fibre dimensions, whereas the 'fuzzy' regions are clumps of nanofibrils released from the fibre walls (modified from Gane et al. 2020_copyright held by same authors as this paper)

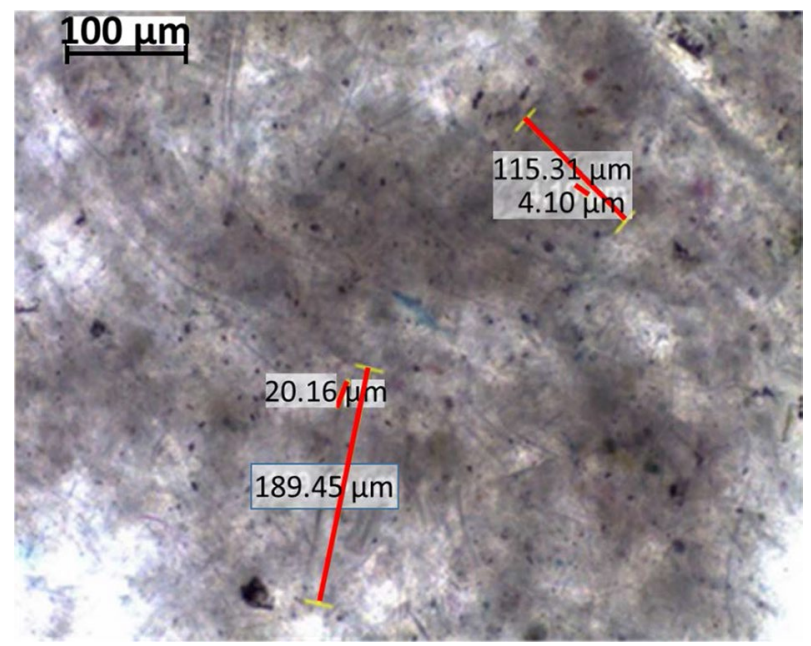




\subsubsection{Ground Calcium Carbonate (GCC)}

Marble and limestone exhibit the rhombohedral calcitic crystal form. The GCC was produced from Norwegian marble, wet ground (Covercarb $^{\circledR}$ 60, Omya Hustadmarmor AS, Molde, Norway), having nominally individual dispersed particle size distribution of 60 $\mathrm{w} / \mathrm{w} \%<1 \mu \mathrm{m}$ and $95 \mathrm{w} / \mathrm{w} \%<2 \mu \mathrm{m}$, with a zeta potential of $\zeta=-27.13 \mathrm{mV}$, at $\mathrm{pH} 9.2$. Though less important in this context, the product was extremely pure and of high brightness, as it has an ISO brightness $\geq 96 \%$. A scanning electron microscope (SEM) image of the fine ground material is shown in Fig. 4. The GCC is produced chemical-free-needed to promote the surface reactivity-so that when in aqueous suspension the particles become flocculated, a property of advantage in this application due to the increased porosity and permeability this creates in the dried coating (also visible in Fig. 4).

The surface characteristics of the GCC particles are summarised in Table 2. The specific surface area $\left(S_{\mathrm{p}}\right)$ was calculated from the linear part of the nitrogen adsorption isotherms (BET) (Brunauer et al. 1938), displaying total pore volume $\left(V_{\text {tot }}\right)$ given at a relative pressure of $p / p_{0}=0.998$. The volume of mesopores $\left(V_{\text {meso }}\right)$ was calculated according to the Barrett, Joyner, and Halenda (BJH) method (Barrett et al. 1951) applied to the desorption branch of the isotherm, and the volume of micropores $\left(V_{\text {micro }}\right)$ calculated from the alpha-S plot (Sing 1998).

\subsubsection{Coating Formulation}

The coating colour was made down (dispersed) using high shear mixing to combine the GCC and MNFC with a ratio of 100 parts by weight (pph) GCC to 10 pph MNFC. Solids content could be adjusted from as low as required by water dilution up to the inherent solids content of the GCC in crumble form $(85-90 \mathrm{w} / \mathrm{w} \%)$ with the low solids content MNFC to provide a maximum of $\sim 75 \mathrm{w} / \mathrm{w} \%$ solids, such that, depending on coating technique and the rheological flow demands, the coating colour can easily be optimised.

Further optimisation of coating structure to aid permeation can be made using a secondary inert particulate mineral spacer. An ideal candidate for this particle packing spacing action is expanded perlite, a volcanic glassy mineral with high porosity and surface area. We used a commercial product Omyasphere ${ }^{\circledR} 120$ (Omya International AG, Baslerstrasse

Fig. 4 SEM image of GCC particles (image taken on a JEM2200FS; JEOL Ltd., Akashima, Tokyo, Japan)

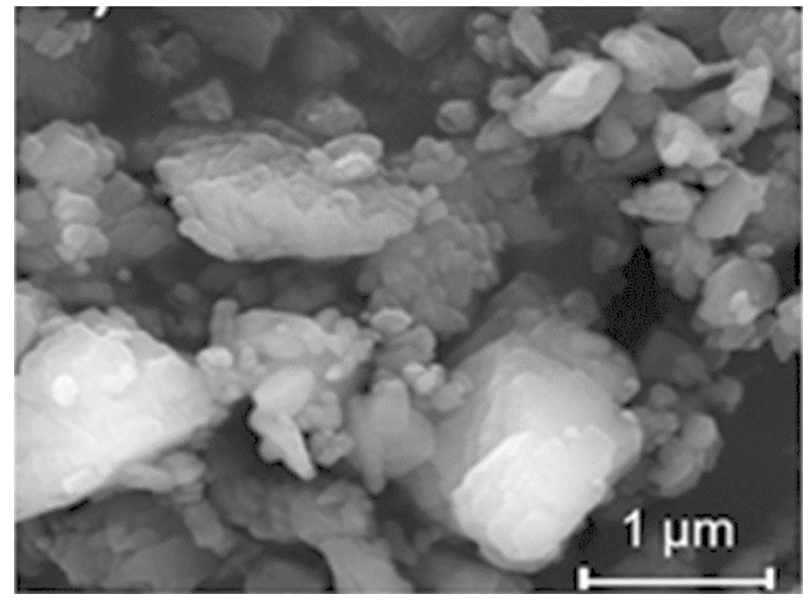




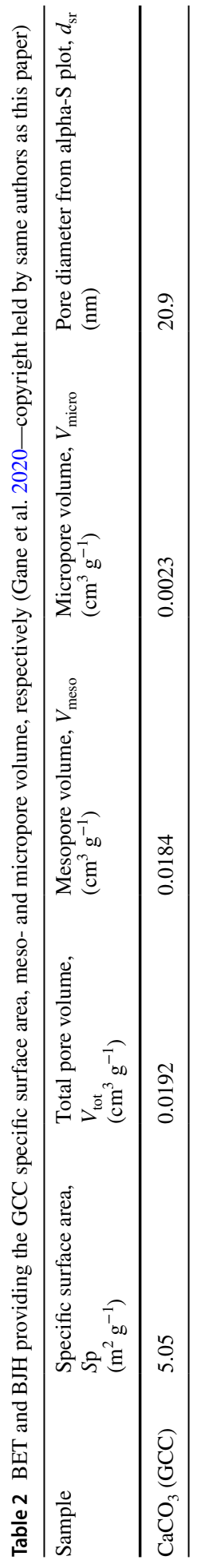


42, 4665 Oftringen, Switzerland). It is a relatively coarse material compared with the GCC, having $98 \mathrm{w} / \mathrm{w} \%$ of particles $\leq 200 \mu \mathrm{m}$. Two addition levels were used, namely, 5:95 pph and 10:90 pph by weight perlite:GCC, respectively.

For completeness, the three coating formulations and their sample nomenclature are shown in Table 3.

\subsubsection{Coating Application}

The target in this work is to devise a filter that can be used either in a permeable throughflow or, preferably for energy reasons, exposed adopting a surface-flow contact. Therefore, it is paramount to maximise the contact possibility between the pollutant gas-carrying air and the active coating. Therefore, to consider a high-quality thick coating, i.e. following the coating industry standard, is both unsuitable for through-flow, due to its impermeable nature and produced surface smoothness, and for surface flow, as it is prone to establishing a static (stagnant) boundary layer.

A convenient way to present large interactive surface is to "print" a patterned, pixelated coating consisting of a spatial distribution of coating over the surface of the permeable substrate. Such a configuration aids both through-flow and surface flow, reducing also the build-up of a static boundary layer. The particle-particle flocculated coating structure placed in this way on the surface, delivers increased permeability with open and highly connected pores for pressure-driven ingress and rapid diffusion of gas.

We achieved this patterned coating distribution, following the method described by Gane et al. (2020), adopting pin/needle coating, by which an array of protruding pins is loaded with coating colour by dipping into a colour reservoir and so transferred to the substrate surface. A region of printed coating (smooth areas) surrounded by uncoated substrate (bare paper fibres) is shown in the SEM image, Fig. 5. The boundary between the printed coating and the substrate is ragged, increasing contact surface and developing high permeability to take advantage of gas transit through the highly permeable substrate.

To illustrate the benefits of pixelated, patterned coating, comparable smooth continuous layer coatings were also prepared using a rod draw-down coater (K Control coater, RK PrintCoat Instruments Ltd, Litlington, Royston, UK).

\subsubsection{Coating Pore Structure and Permeation Analysis}

Techniques have been developed previously to perform mercury intrusion and liquid permeability evaluations of particulate coating colours based on fabricating macroscopic tablets from the coating colour slurry (Schoelkopf et al. 2000; Ridgway et al. 2003). By creating a large-scale replica of the coating layer in this way, it is possible to obtain sufficient realistically formed, particle packed and dried material to provide reliable pore volume

Table 3 Coating formulation proportions and sample labelling

\begin{tabular}{llll}
\hline Coating constituent & GCC & GCC 5P & GCC 10P \\
\hline GCC/pph by weight & 100 & 95 & 90 \\
Perlite/pph by weight & 0 & 5 & 10 \\
MNFC/pph by weight on & 10 & 10 & 10 \\
100 parts mineral & & & \\
\hline
\end{tabular}




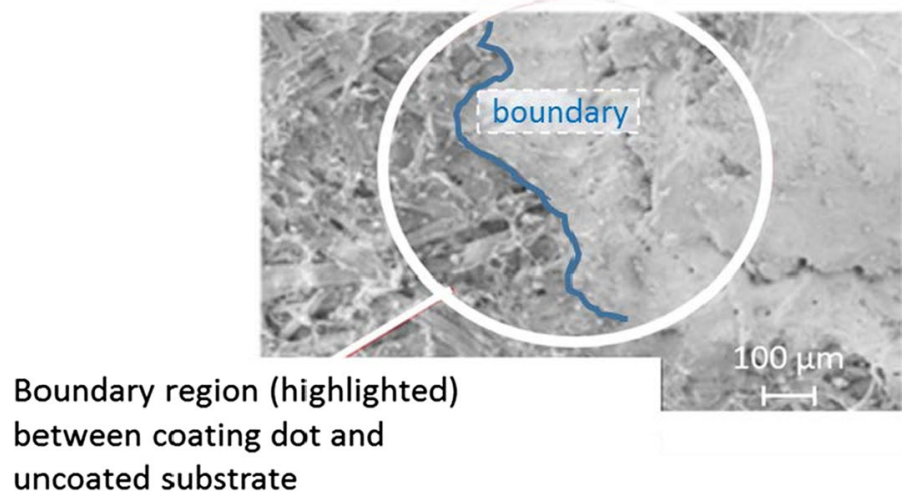

Fig. 5 SEM image of the boundary region (highlighted and shown by the blue line) between the active printed dot to the right and the uncoated porous substrate to the left (image taken on a JEM-2200FS; JEOL Ltd., Akashima, Tokyo, Japan): (modified from Gane et al. 2020_copyright held by same authors as this paper)

data, and to be able to saturate and permeate inert liquid under pressure through the tablet structure.

Field emission SEM (FESEM) images of sections of sample tablets made from the three coating recipes show the packing structure of the particles, Fig. 6 . The differences between the fine GCC, Fig. 6a, and the large, almost broken ceramic pottery-like particles of the expanded perlite, Fig. 6b, are clearly visible. The action of adding perlite generates a completely different more porous packing of the particles in the coating structure. Also, the larger microfibrillar fraction in the MNFC, acting as binder, can be readily discerned.

Mercury intrusion was performed on a section of tablet from each of the three coating formulations using an Autopore V porosimeter (Micromeritics, Norcross, GA, USA). The intrusion pressure applied spanned a range up to $414 \mathrm{MPa}$, equivalent to a Laplace throat diameter of $0.004 \mu \mathrm{m}(\sim \mathrm{nm})$. An equilibration time of $20 \mathrm{~s}$ was used at each pressure. Artefacts occurring at high intrusion pressure due to skeletal material compressionin this case mainly the organic MNFC binder/humectant component-are removed using an applied correction to eliminate the elastic behaviour seen during the initial region of decompression, where normally extrusion hysteresis would occur. The erroneous apparent intrusion in this region due to compression otherwise would falsely indicate the presence of non-existent ultrafine pores (Gane et al. 1996). The data correction for this effect is made using Pore-Comp (software module in the PoreXpert suite for pore structure modelling and analysis) for mercury and penetrometer effects and for elastic sample compression, where applicable (Gane et al. 1996).

Experimental liquid permeation was performed using a cuboid piece of sample tablet $\left(\sim 1.5 \times 1.5 \times 1.0 \mathrm{~cm}^{3}\right)$ prepared by placing into a cylindrical mould and resin poured around it, Fig. 7a. The quickly rising viscosity of the chosen curing resin results in a limited penetration of approximately 1-2 mm locally at the outer boundaries of the sample. This resin penetration depth is clearly visible because of the opacity change at the edge of the sample and can, therefore, be calibrated. The open area of the porous sample, i.e. that free from resin, is evaluated so that the permeable cross-sectional area can be established. The sample discs are placed in a dish containing the probe liquid in order to saturate the void network of the sample before placing in the apparatus. Hexadecane, density, 


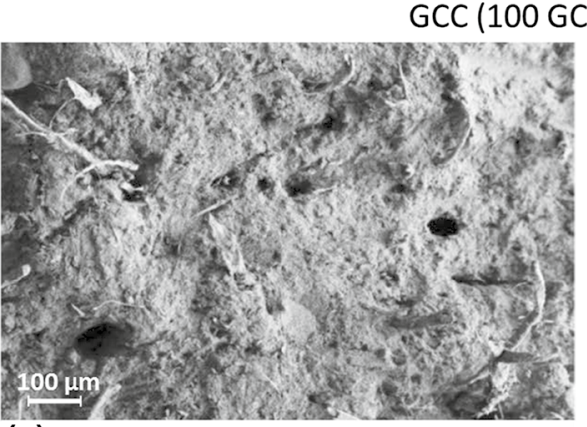

(a)
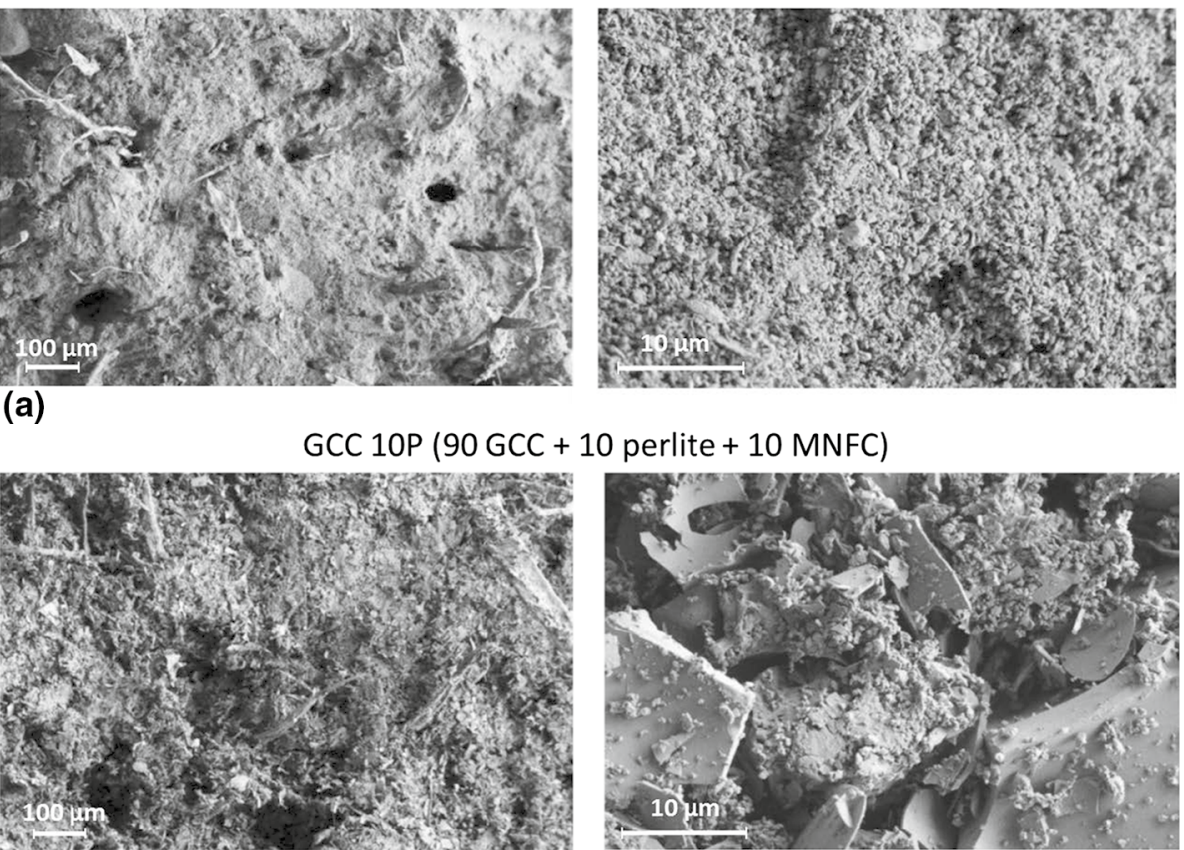

(b)

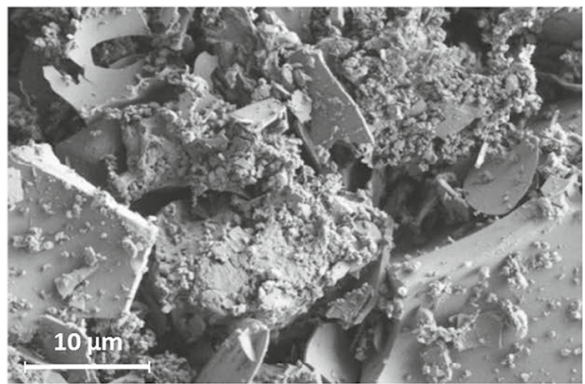

Fig. 6 Micrographs by field emission-scanning electron microscope (FESEM, Zeiss Sigma VP, White Plains, NY 10601, USA) using secondary electron detector (SE) to show particle shape of the samples: a GCC and b GCC 10P

\section{Cuboid section of tablet}

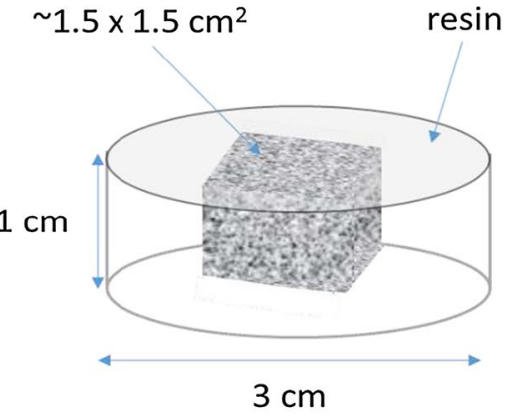

(a)

(b)

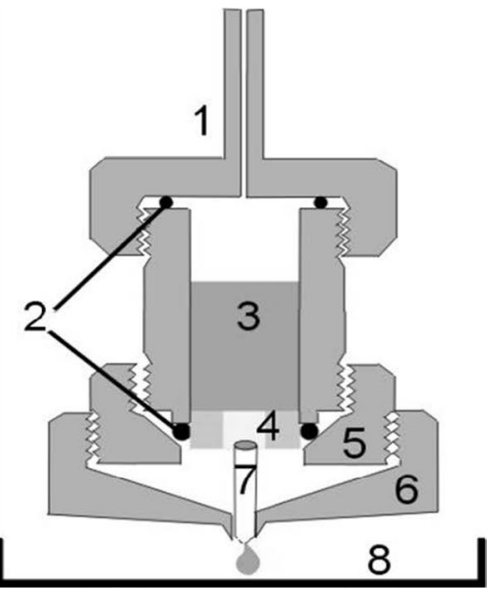

Fig. 7 a Preparation of tablet cuboid piece for permeability measurement, b permeability measurement cell: (1) lid with pressure inlet, (2) sealing O-rings, (3) liquid cell; outer diameter $=40 \mathrm{~mm}$, (4) porous sample embedded in resin disc of diameter $=30 \mathrm{~mm}$, (5) fixing ring compresses the O-ring which seals the resin disc, (6) security shroud and drop collector, (7) drop captor (Teflon tubelet), (8) dish on microbalance 
$\rho=773 \mathrm{~kg} \mathrm{~m}^{-3}$, and viscosity, $\eta=0.0034 \mathrm{~Pa} \mathrm{~s}\left(\mathrm{~kg} \mathrm{~m}^{-1} \mathrm{~s}^{-1}\right)$, is used in the experiments due its inert behaviour in respect to otherwise water-sorbing materials, such as MNFC.

The sample disc is then placed in a specially constructed pressure cell (Ridgway et al. 2003). The cell design used for the pressurised permeability experiments is shown schematically in Fig. $7 b$.

The use of the resin to embed the samples allows for rigid clamping and sealing of the sample into the pressure cell chamber. Gas over-pressure is supplied from a nitrogen bottle. The pressure cell is fixed over a microbalance and a PC samples the balance data. A drop captor device is needed in the base of the cell to guide the permeated liquid drops to the outlet. An important point of practical technique is that the whole chamber below the position of the sample has to be pre-wetted with the liquid so that each drop leaving the sample causes a drop to fall into the sampling dish. Once these precautions are taken, the continuity of flow is ensured.

The continuous laminar flow can be expressed in terms of the Darcy permeability constant, $k\left(\mathrm{~m}^{2}\right)$, as

$$
\frac{\mathrm{d} V(t) / \mathrm{d} t}{A}=\frac{-k \Delta P}{\eta l}
$$

where $(\mathrm{d} V(t) / \mathrm{d} t) / A$ is defined as the flux or volume flow rate per unit cross-sectional area, $A, \Delta P$ is the applied pressure difference across the sample, $\eta$ is the viscosity of the liquid and $l$ is the length of the sample, i.e. macroscopic path length of the permeation.

\subsubsection{Gas Exposure}

The coated filters were first pleated and scrolled and then placed into a gas pressure flow meter chamber as shown in the schematic in Fig. 8. The concentration and flow rate of $\mathrm{NO}_{2}$ gas-in-air (supplied by Messer-Gases for Life, Serbia) was controlled and recorded using a mass flow controller (ZF-MFC 1 series, TK-Fujikin Corporation, Busan, Korea). The exposure time used was $4 \mathrm{~h}$ for each filter construct.

The $\mathrm{NO}_{2}$ molar gas-in-air flow rate and concentration are shown in Table 4 .

The amount of sorbed $\mathrm{NO}_{2}$ was back-calculated stoichiometrically on the basis of nitrite and nitrate ion concentration formed during exposure to $\mathrm{NO}_{2}$, measured by extraction from the filter after exposure using ion chromatography (DionexTM DX-300; Thermo Fisher Scientific Inc., Waltham, MA, USA).

\section{Experimental Results}

The focus here is on comparing the performance of a porous cellulose fibre substrate pincoated (patterned, pixelated) with a gas-sorbing active coating versus a continuous active coated layer. The two filters were either pin-coated on two sides or continuous coated on one side for comparison. The choice to coat only one side of the filter with the continuous layer was made because a second coating on the opposite side, having very low permeability, would have hindered gas from passing through the porous substrate contacting the underside of the single layer of active coating, thus rendering the continuous coated layer filter even more inefficient in this comparison. 


\section{Pleated surface flow coated filter}

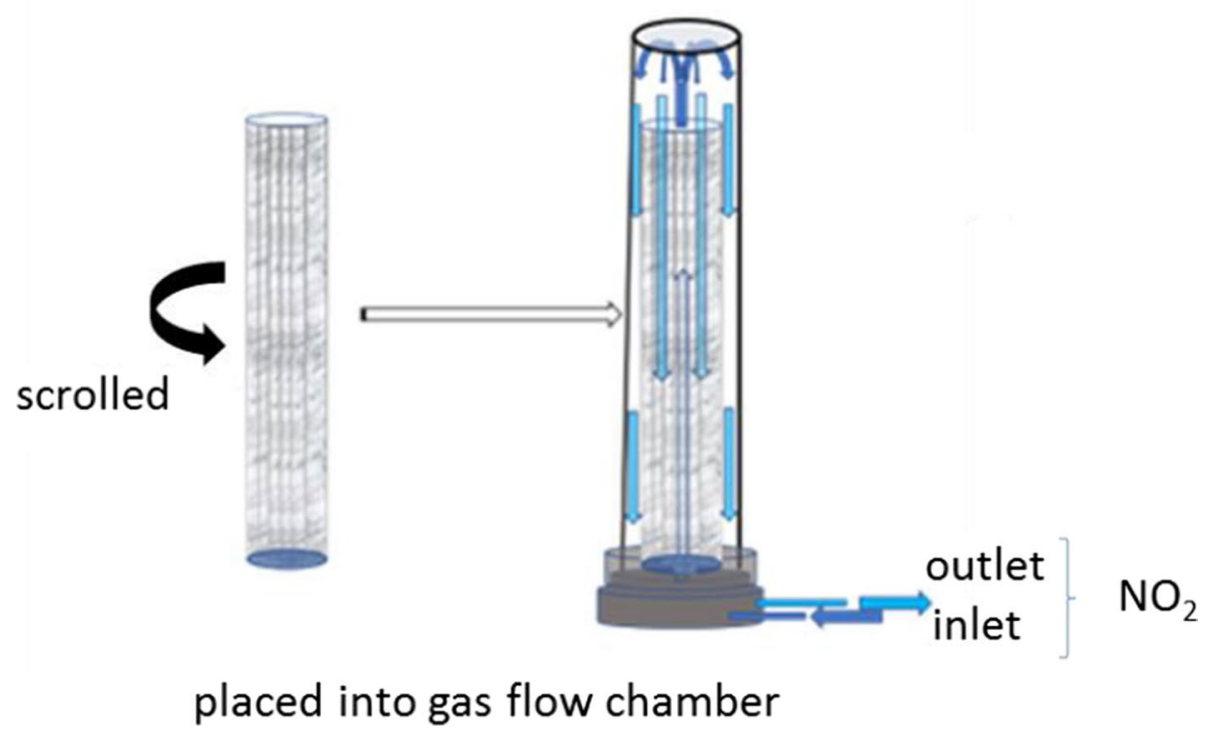

Fig. 8 Sorption of $\mathrm{NO}_{2}$ from air onto filter using a gas flow meter chamber

Table $4 \quad \mathrm{NO}_{2}$ gas-in-air volume flow rate, $\mathrm{NO}_{2}$ concentration, and molar flow rate

\begin{tabular}{lll}
\hline $\begin{array}{l}\text { Flow rate, } v \\
\left(10^{-6} \mathrm{~m}^{3} \mathrm{~s}^{-1}\right)\end{array}$ & $\begin{array}{l}\mathrm{NO}_{2} \text { molar volumetric concentration } \\
\left(10^{-5} \mathrm{~mol} \mathrm{~m}^{-3}\right)\end{array}$ & $\begin{array}{l}\mathrm{NO}_{2} \text { molar flow rate } \\
\left(10^{-10} \mathrm{~mol} \mathrm{~s}^{-1}\right)\end{array}$ \\
\hline 3.33 & 6.304 & 2.099 \\
\hline
\end{tabular}

\subsection{Gas Sorption onto Filter}

Results of $\mathrm{NO}_{2}$ sorption from an $\mathrm{NO}_{2}$-in-air synthetic mix flowing over the respective filter surfaces is shown in Table 5. Clearly, the pixelated coating displays the significantly more efficient exposure of the active coating to the gas.

The challenge now is to quantify the dominant gas transport mechanisms at play in order to establish the functional behaviour of the distribution of the mineral active coating on the fibrous substrate. Understanding the coating pore structure is key.

\subsection{Pore Size Distribution and Structure Permeability}

\subsubsection{Porosimetry}

The results of mercury intrusion porosimetry are shown in Fig. 9 and the porosity values of the coatings are shown in Table 6. 


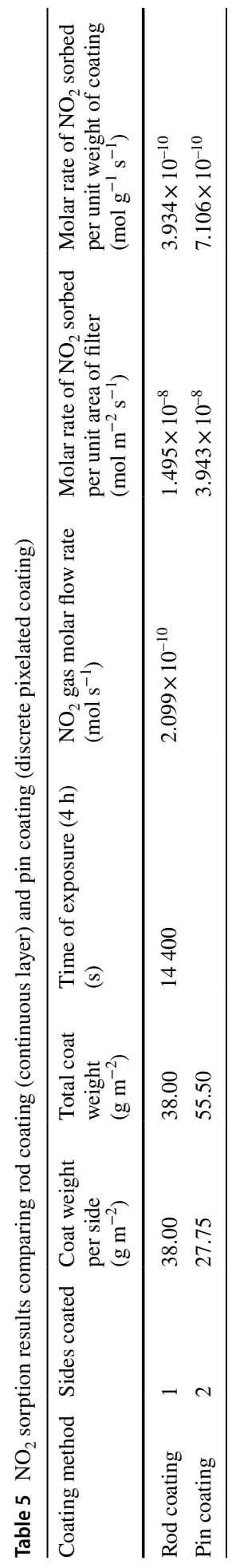



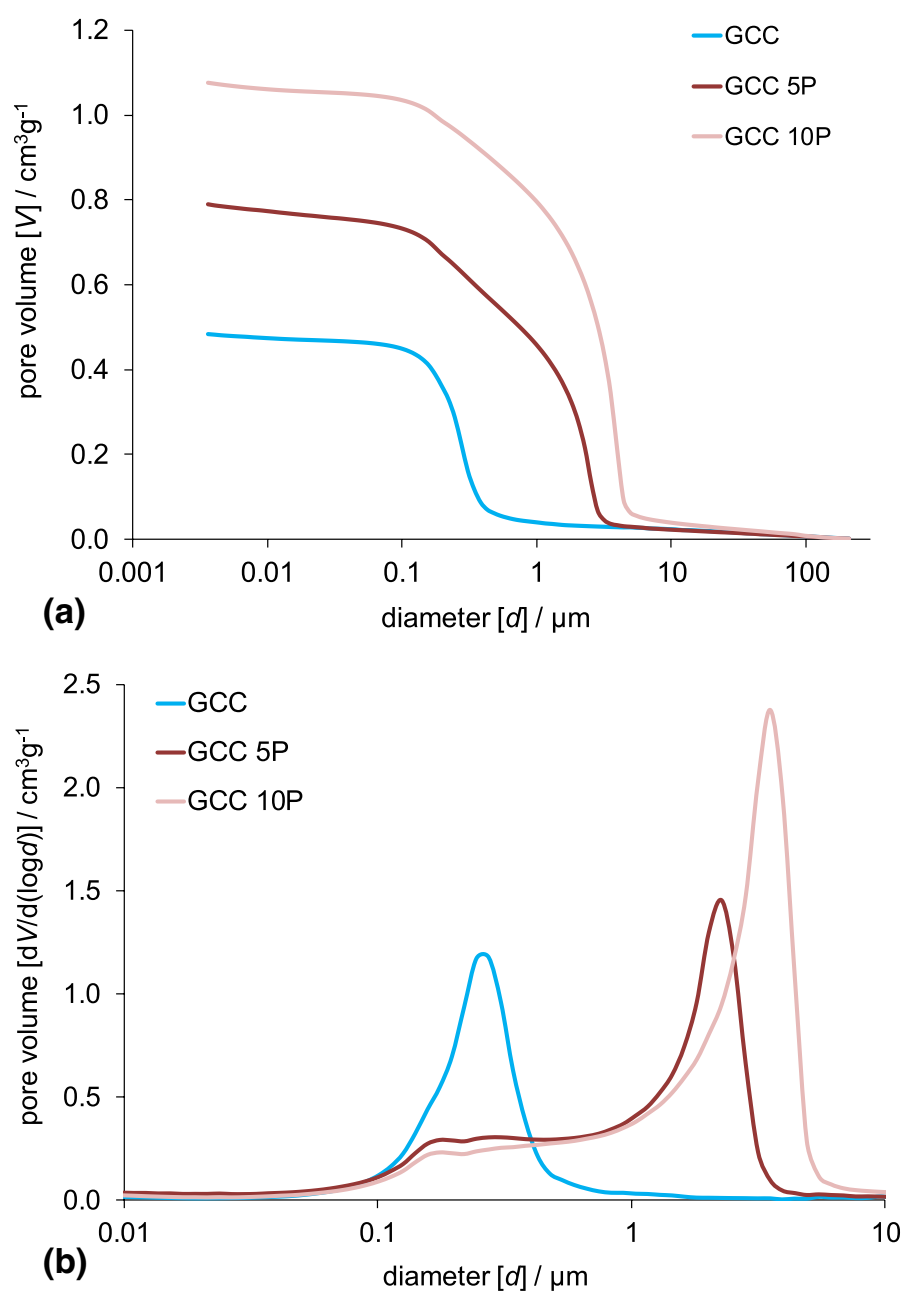

Fig. 9 Mercury intrusion: a cumulative intrusion volume, b differential pore volume distribution based on pore entry diameter

Table 6 Sample specific pore volume and porosity

\begin{tabular}{lll}
\hline Sample & $\begin{array}{l}\text { Total specific intruded volume } \\
\left(\mathrm{cm}^{3} \mathrm{~g}^{-1}\right)\end{array}$ & Porosity, $\phi(\%)$ \\
\hline GCC & 0.48 & 54 \\
GCC 5P & 0.79 & 64 \\
GCC 10P & 1.08 & 71 \\
\hline
\end{tabular}

The mercury intrusion data in Fig. 9 are indicative of the packing structure and the general fine pore structure of the tableted coatings. The main peak lies at $0.27 \mu \mathrm{m}$ for the sample containing only GCC and MNFC (sample GCC). The finest peak in this large pore region represents a pore diameter of $2.3 \mu \mathrm{m}$ for the sample with $5 \mathrm{pph}$ perlite 
(GCC 5P), and the highest peak at the larger pore diameter of $3.5 \mu \mathrm{m}$ is for the sample with 10 pph perlite (GCC 10P). The change from monomodal intrusion to trimodal on the addition of perlite is readily seen in Fig. $9 \mathrm{~b}$.

In the finer pore region, the high peak for the GCC formulation as single pigment is progressively replaced by the internal pore volume and pore packing of the additive mineral, which has disrupted the close packing of the GCC. In contrast to the solid nonporous GCC particles, the added expanded perlite material is itself highly porous. The peaks in this case are, therefore, trimodal, representing the finest internal pores (intraparticle volume) and less fine interparticle pores, together with any retained carbonate packing pore volume. The more additive pigment is used, the more the packing changes towards the additive mineral pore structure in this region as the remaining carbonate contribution decreases further. Thus, the greater the level of additive mineral, the less the influence of the remaining carbonate pore volume, and so the overall pore volume in this region decreases despite the intrinsic increase in internal pore volume provided by the additive.

There are smaller peaks towards the finer pore sizes, likely representing fibril/pigment interaction of the pore structure. This region is shown in more detail in Fig. 10.

The peaks for the two samples including perlite in this fine pore region appear bimodal, leading to the overall trimodality of the complete pore size distribution, Fig. 10, having their pore sizes at $0.2 \mu \mathrm{m}$ and $0.3 \mu \mathrm{m}$, respectively. The peaks in this region are lowest for the sample with the highest perlite loading, as discussed above, due to the greater displacement of carbonate-carbonate interparticle pore packing. It is, therefore, important to understand this relationship as, intuitively, without consideration of the replacement of carbonate-carbonate packing it would be erroneously assumed that relative fine pore volume would otherwise increase as addition levels are increased, given the relatively large pore surface area porosity introduced using perlite.

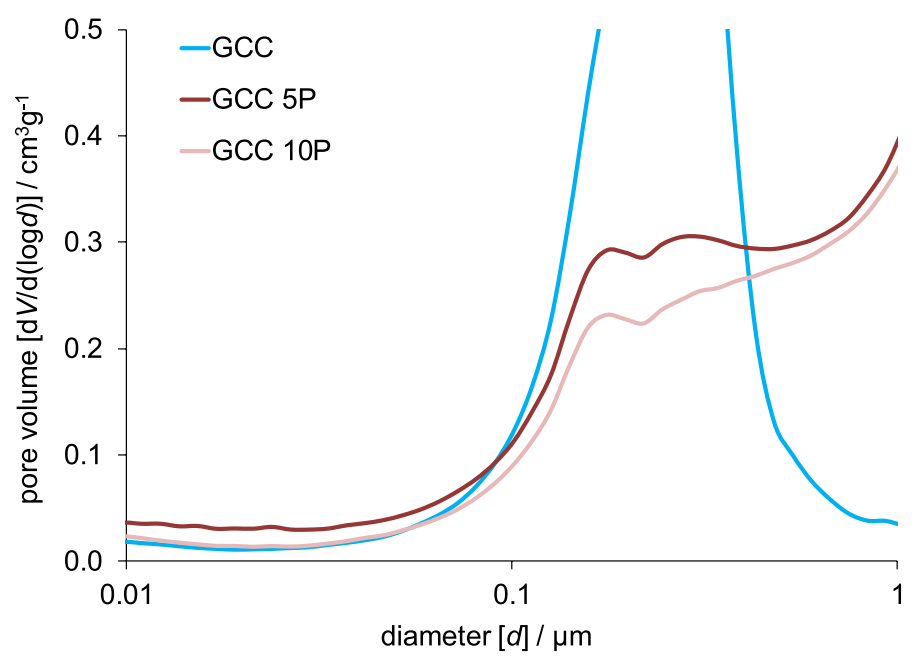

Fig. 10 Focusing-in on the fine pore size distributions by expanding the $y$-axis scale for the pore diameter region $0.01-1 \mu \mathrm{m}$ to show the fine-scale data for samples GCC 5P and GCC 10P. [Please note that the peak of the GCC alone has been truncated to maintain the focus on the fine pore structure of the blends-if desired it can be clearly studied for comparison in Fig. 9.] 


\subsubsection{Liquid Permeability}

The samples were left for $\sim 1 \mathrm{~h}$ to saturate with hexadecane before the permeability measurements were performed. Permeation through the saturated structure was then left to run for $5 \mathrm{~min}$, such that enough data were collected to fit a linear flow-through (flux) behaviour. Due to relatively large volume flow, a single measurement sufficed to show the expected increase in permeability as perlite addition increases.

The liquid permeation results for the samples are shown in Table 7.

The permeability values follow the trend of the main large pore size peak in the pore diameter distribution, Fig. 9b, with the sample GCC, which had the finest measured pore diameter, showing the lowest permeability, and sample GCC 10P having the highest permeability.

\section{Modelling}

Having measured the liquid permeability experimentally, we modelled the pore structure to ascertain the relative flux difference between liquid and gas under pressure-driven permeation, and to establish a model tortuosity for the sample. Modelling the gas flow over the surface structure is then considered to establish the likely pressure drop experienced resulting from the aerofoil (Bernoulli) effect as gas flows over the printed coating dot. Comparison is then made between the pressure-driven permeation and the diffusive gas transport into the structure, the latter assuming maximum transport towards an infinite sorption sink, providing a mechanism of excess sorption capacity.

\subsection{Pore structure Modelling}

The PoreXpert modelling suite was used to simulate the mercury intrusion behaviour. The model represents the void structure of a porous medium as a series of identical interconnected unit cells with periodic boundary conditions (Laudone et al. 2005, 2008). Void shapes are simplified to cubic pores, positioned with their centres at each array node, connected by up to six cylindrical throats in each orthogonal Cartesian direction. The simplification of the void shapes does not greatly affect the percolation or diffusion properties, which depend mainly on those properties which are quantitatively matched to experiment, namely the volume, cross-sectional area, and connectivity of the voids.

The void network modelling methodology is described in detail in Levy et al. (2015). The characteristics of the unit cell are adjusted using an eight-dimensional Boltzmannannealed amoeboid simplex, which searches within the multi-parameter space for an optimum void network that matches closely the experimentally measured porosity and, in this

Table 7 Experimental liquid permeability values of the samples derived applying Eq. (2)

\begin{tabular}{ll}
\hline Sample & $\begin{array}{l}\text { Darcy perme- } \\
\text { ability coefficient, } k \\
\left(10^{-15} \mathrm{~m}^{2}\right)\end{array}$ \\
\hline GCC & 0.80 \\
GCC 5P & 6.38 \\
GCC 10P & 23.14 \\
\hline
\end{tabular}


case, pressure-driven intrusion characteristics of the porous medium. The five parameters defining the parameter space are throat skew, throat spread, pore skew, connectivity, and correlation level (Price et al. 2009). Throat spread and throat skew define the variance and asymmetry of the throat size distribution, respectively. The distribution follows an Euler beta function, which encompasses Gaussian-like and Poisson-like types, as well as bimodal distributions with most void sizes at close to the minimum pore diameter, $d_{\text {pore-min }}$, and the maximum pore diameter, $d_{\text {pore-max }}$. The pore skew parameter bulks up the volume of pores and compensates for the difficulty of achieving, in a regularly spaced, and hence inefficiently packed matrix of void features, a value of porosity matching the value obtained experimentally. The representation of this effect in the resulting unit cell is that there are larger numbers of pores at the maximum size of the distribution. These could be individual pores, but are more likely to be clusters of pores which are not differentiated by the mercury porosimetry, because they comprise a single narrow throat allowing entry into a cluster of pores and throats all with dimensions greater than the single narrow throat, i.e. the well-known phenomenon of so-called pore shielding. There are also three Boolean parameters: namely whether the network is fully connected, whether the experimental porosity is achieved, and whether all the void features are separate in space without geometric overlap. Permeability is strongly related to the connectivity of larger pores. Connectivity in the model is defined as the average of the local connectivities between neighbouring voids averaged over the entire sample, or, in this case, over the entire representation of the sample, i.e. over a single unit cell.

The void network structure resulting from such an optimisation procedure is not unique, so five stochastic realisations were generated which match the experimental intrusion characteristics. The most representative simulated structure for each sample was then found by choosing the realisation for which the least number of fitting parameters had their respective deviation from the mean of the complete parameter set larger than the standard deviation of that model set. If more than one sample model had met this criterion, then the model with all parameters closest to the mean would have been chosen, but amongst these samples, this selection was not necessary. The model chosen represents a random structure. It was not attempted to generate the fine subtleties of the experimental trimodal pore size distribution. In principle, layered structures and centred distribution structures are possible using the PoreXpert suite. However, the main function of interest here is permeability, and permeation under external pressure is dominated by fluid passage through the larger high connectivity connecting throats, such that fine structure subtleties are of limited importance, though they are of course extremely important in respect to gas-surface reactivity. Once a representative pore structure was established a simulated permeability for both hexadecane (Darcy model for mineral oil liquid) and nitrogen (Knudsen model for $\mathrm{N}_{2}$ gas) could be calculated. The difference in the calculation for either chosen gas or liquid being the mean free path length in the calculation, obviously greater for the gas.

In the chosen model, each unit cell of side length $h_{\text {cell }}$ comprises an array of $n \times n \times n$ pores, where $h_{\text {cell }}=n \times$ the pore row spacing (node to node distance). For the samples modelled here, $n=15$ and $h_{\text {cell }}=3154 \mu \mathrm{m}$ for sample GCC, $3412 \mu \mathrm{m}$ for GCC 5P, and 3 $268 \mu \mathrm{m}$ for GCC 10P. The PoreXpert fitting parameters, the simulated permeability values, and tortuosity (Laudone et al. 2005) are shown in Table 8.

The correspondence of model and experimental data is shown for each structure in Fig. 11a-c, together with the model structure (d), respectively. Viewing the pore model structures in (d) shows the increasing porosity moving from GCC through GCC 10P, noting that the coloured cubes indicate the pore space and the uncoloured regions the solid phase, i.e. the more continuous the coloured cube structure the more porosity it has. Of interest 


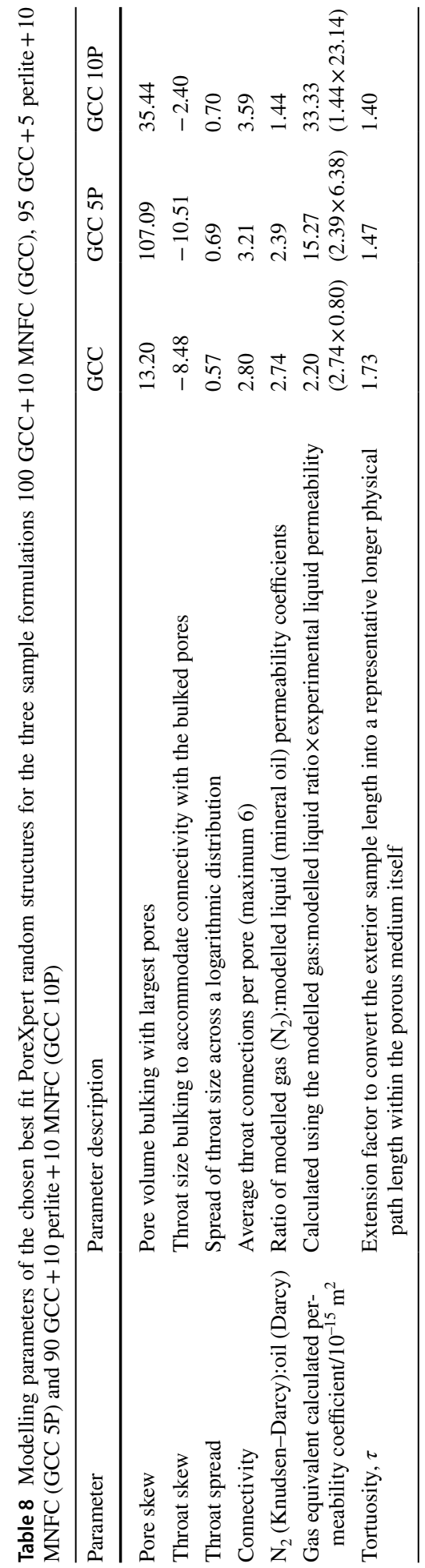



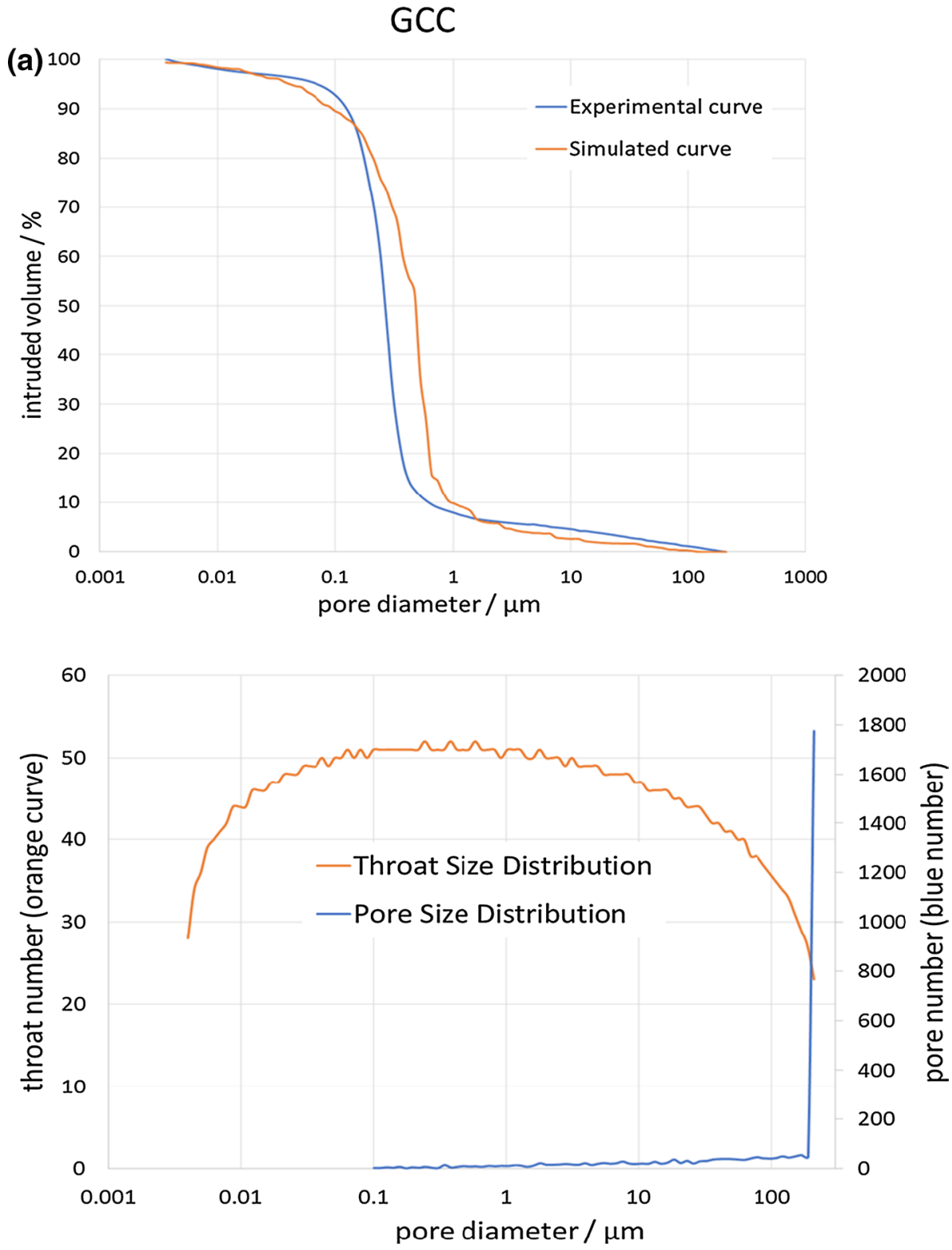

Fig. 11 Comparison of model intrusion curves with the experimental data a GCC, b GCC 5P, c GCC 10P, and $\mathbf{d}$ the pore model structures

also is the way the model captures the change from the tight GCC packing, with the concentration of throat size in the middle of the throat size distribution, Fig. 11a, to the loose packing generated by the addition of large particle size expanded perlite at 5 pph (GCC5P) but with further dominating fine-scale internal pores represented by the increased occupation of fine and large throats in the model throat size distributions described in, Fig. 11b, c. 


\section{GCC 5P}
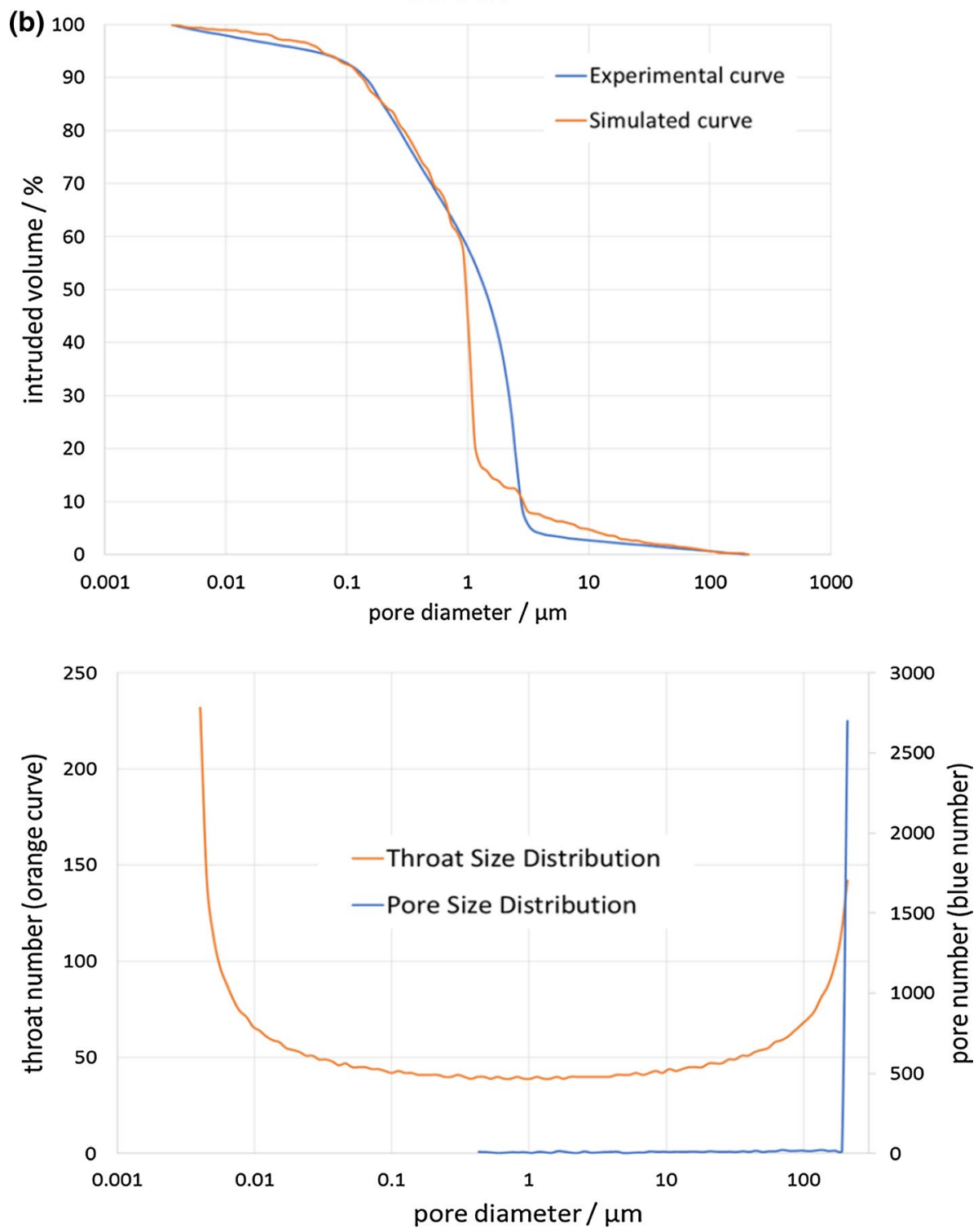

Fig. 11 (continued)

At the same time, as discussed in respect to the mercury porosimetry Fig. 9, the disruptive effect of the added perlite creating increased porosity is clearly seen in the model structures shown in Fig. 11d, transitioning from GCC to GCC5P and finally GCC10P.

It is stressed in this work that porosity alone does not determine permeability, the latter being the important parameter here for gas transport, where permeability is determined as a combined effect of porosity and connectivity between the pores. Included in 

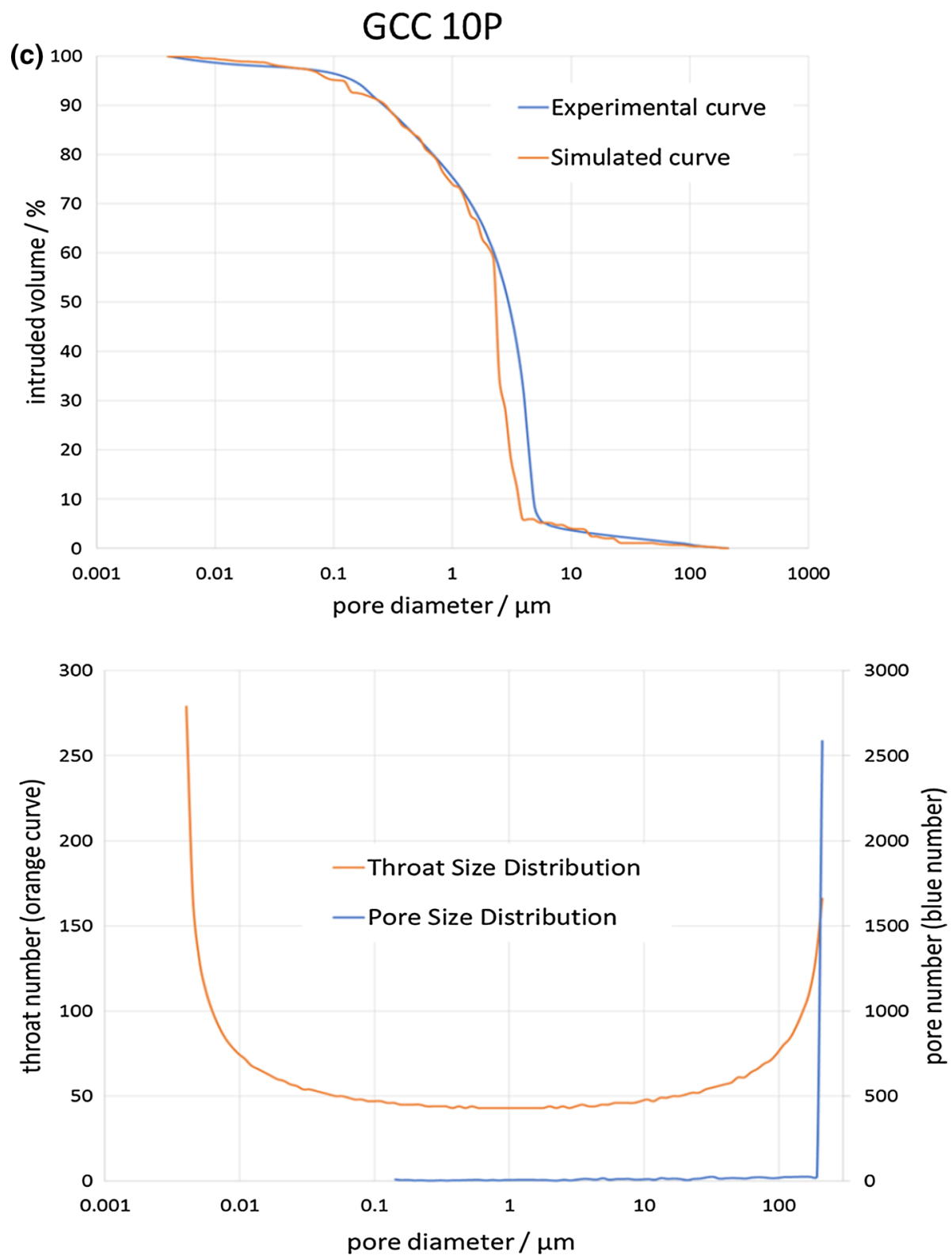

Fig. 11 (continued) 

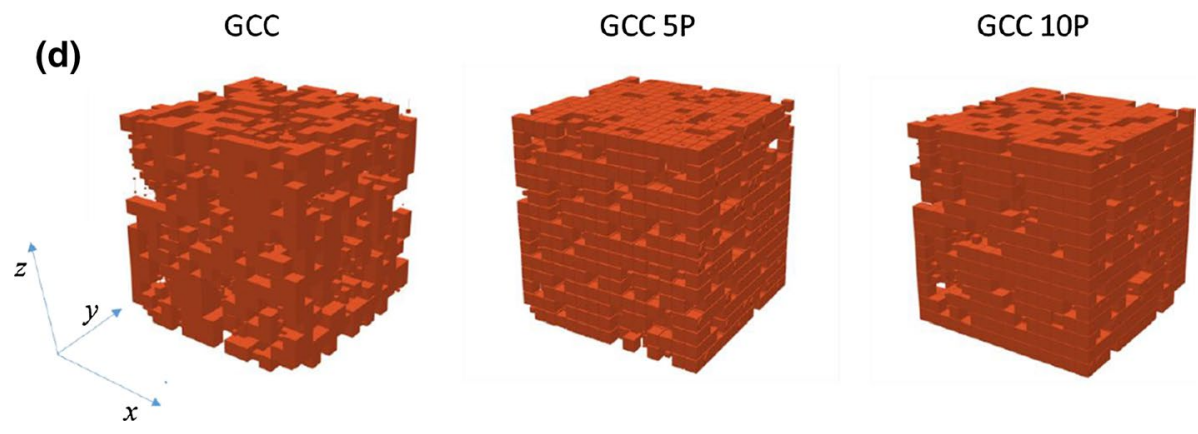

Fig. 11 (continued)

Table 8 are the values for the gas permeability calculated by taking the modelled ratio between gas and liquid permeability and using this as a multiplicative conversion factor to calculate gas permeability from measured liquid permeability for the experimental coatings.

\subsection{Gas Diffusion}

A single gas diffusion rate through an unobstructed medium can be calculated assuming an infinite sink for the sorption of the gas. The volume rate of diffusive flow, $F$ [equivalent to $\mathrm{d} V / \mathrm{d} t$ as expressed for liquid in Eq. (2)] into an idealised continuous cylindrically symmetrical pipe of length $l$, radius $r$, and cross section $A\left(=\pi r^{2}\right)$ is given by

$$
F=\frac{A D_{0}}{l}
$$

where $D_{0}\left(\mathrm{~m}^{2} \mathrm{~s}^{-1}\right)$ is the diffusion coefficient of the gas in free space (Cape 2005).

In the case considered here, the medium is not unobstructed but is a pore network with solid material defining the tortuous path the gas must follow through the void structure. Furthermore, the diffusion coefficient $D_{0}$ becomes that of $\mathrm{NO}_{2}$ in air, $D_{\mathrm{NO} 2}$ in air. Palmes and Tomczyk (1979) give a diffusion coefficient value for $\mathrm{NO}_{2}$ in air as $1.54 \times 10^{-5} \mathrm{~m}^{2} \mathrm{~s}^{-1}$ at $21.1^{\circ} \mathrm{C}$ and 1 bar (atmospheric) pressure.

Within the pore network structure, the physical cross-sectional area of the sample, $A_{\text {sample }}$, must be corrected for the accessible space, namely the porosity $\phi$, and the physical sample length $l_{\text {sample }}$ by the tortuosity $\tau$, such that Eq. (3) for this tortuosity model $F_{\tau}$ becomes

$$
F_{\tau}=\frac{A_{\text {sample }} \phi D_{\mathrm{NO} 2 \text { in air }}}{l_{\text {sample }} \tau}
$$

To generalise the study of gas diffusion into this type of porous structure medium, we apply continuum percolation considering the air-filled porosity and its critical value for percolation in the porous medium, respectively (Berkowitz and Balberg 1993; Hunt 2001; Hunt and Ewing 2003, 2009). Since, in practice, gas diffusion is only measured above the 
percolation threshold, Ghanbarian and Hunt (2014) showed that gas diffusion in a continuum porous medium could be represented by the universal scaling law

$$
\frac{D_{\mathrm{p}}}{D_{0}}=\left(\frac{\varepsilon_{\mathrm{air}}-\varepsilon_{\mathrm{t}}}{1-\varepsilon_{\mathrm{t}}}\right)^{\mu}
$$

where $\varepsilon_{\text {air }}$ is the air-filled porosity, which we assume for these highly connected pores is the same as the mercury intruded porosity $\left(\varepsilon_{\text {air }}=\phi\right.$, where $\phi$ is the intruded porosity of the sample), and $\mu$ is considered a fixed exponent $[\mu=1.3$ for $2 \mathrm{D}$ (used here) and $2 \pm 0.02$ for 3D diffusion (Stauffer and Aharony 1994)]. By replacing $D_{0}$ again by $D_{\mathrm{NO} 2}$ in air in Eq. (5), $D_{\mathrm{p}}$ can then be calculated approximately by

$$
D_{\mathrm{p}}=\frac{F_{\tau} l_{\text {sample }}}{A_{\text {sample }}}
$$

The parameter $\varepsilon_{\mathrm{t}}$ can be refined as a fraction of porosity $\left(\varepsilon_{\mathrm{t}}=p \phi\right)$ by comparing diffusion results from this continuum percolation model with those derived from the tortuous path model above, Eqs. (4) and (6), using

$$
p=\frac{\frac{1}{\phi}\left(\frac{D_{\mathrm{p}}}{D_{\mathrm{NO} 2 \text { in air }}}\right)^{\frac{1}{\mu}}-1}{\left(\frac{D_{\mathrm{p}}}{D_{\mathrm{NO} 2 \text { in air }}}\right)^{\frac{1}{\mu}}-1}
$$

In this way, a realistic value for $\varepsilon_{\mathrm{t}}$ can be generated for future use without the need to model the pore structure to derive a tortuosity.

The net flow of the gas by the continuum percolation model diffusion through a physical sample of length $l_{\text {sample }}$ and cross section $A_{\text {sample }}$ is then calculated, once again assuming an infinite sink within the structure according to Eq. (3), by substituting $D_{0}$ by $D_{\mathrm{p}}$

$$
F_{\varepsilon_{\mathrm{t}}} / A_{\text {sample }}=\frac{D_{\mathrm{p}}}{l_{\text {sample }}}
$$

where $F_{\varepsilon t}$ is the volume flow rate by the percolation continuum model, $A_{\text {sample }}$ is the crosssectional area of the sample, and $l_{\text {sample }}$ the sample length through which the diffusion occurs: once again these latter parameters correspond with those expressed in Eq. (2) for the pressure-driven (Darcy) permeation.

\subsection{Gas-Surface Flow}

The geometry of the surface is shown schematically in Fig. 12. The shape of the printed coating dot is formed due to the action of surface tension, and so is assumed to follow that of a segment of a spherical droplet.

Computational fluid dynamics (CFD) modelling of the gas flow over the patterned (pixelated) filter surface was made for a range of surface-flow velocities $\left(5,10\right.$ and $\left.20 \mathrm{~m} \mathrm{~s}^{-1}\right)$ using the COMSOL 2D finite element software (COMSOL Multiphysics ${ }^{\circledR}$ Modelling Software, COMSOL, Inc., 100 District Avenue, Burlington, MA 01803, USA) and compared with that of a perfectly flat surface. It is assumed that the flow regime is turbulent, hence a Reynolds averaged Navier-Stokes (RANS) modelling approach was applied. Furthermore, 
Hexagonal close-packed needle coater pin separation

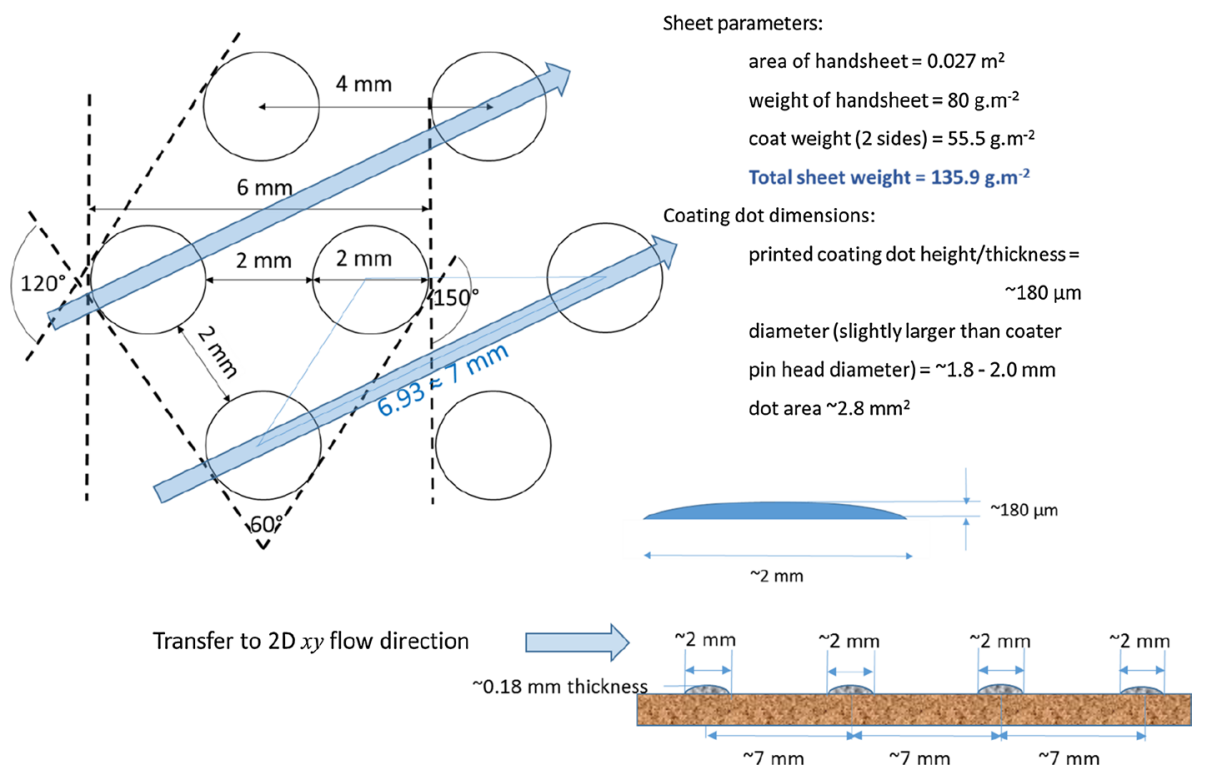

Fig. 12 Geometry of pin-coated surface showing approximation in 2D modelled gas flow direction (blue arrows)

an SST (Menter's shear stress transport) turbulence model was included, which provides a two-equation eddy-viscosity turbulence representation. The model thus combined the $k_{\mathrm{e}^{-}}$ $\omega$ turbulence model used in the region close to the boundary (Menter 1994), where $k_{\mathrm{e}}$ is the kinetic energy of the turbulence and $\omega$ the dissipation of that energy, thus identifying

Mesh - close to wall-pin dot

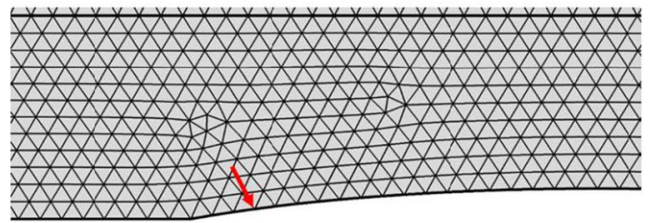

Mesh - bulk liquid

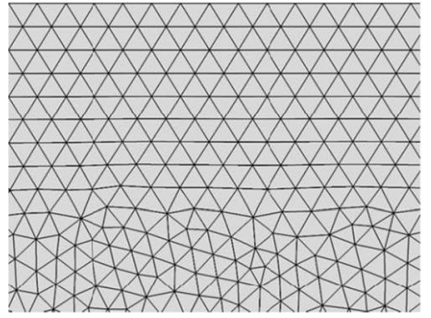

\section{Mesh statistics}

\section{Complete mesh}

Triangular elements:

Edge elements:

Vertex elements:

\section{Domain elements}

Number of elements:

34011

Minimum element quality:

0.7308

Average element quality:

0.9813

Mesh area:

$139.1 \mathrm{~mm}^{2}$

Maximum growth rate:

2.007

Average growth rate:

Fig. 13 COMSOL 2D finite element mesh construction and mesh statistics 
particularly pressure gradients, they being the focus of this work, and the $k_{\mathrm{e}}-\varepsilon$ in the free shear flow (Launder and Sharma 1974), which is also a kinetic energy-based model but applies a mixing length, $\varepsilon$.

The fluid domain was split into two subdomains to decrease computational time by using different mesh scales. This technique enabled more accurate results to be generated where most needed, i.e. for the flow over the curved coating spot surfaces and under adverse flow gradients (Tabataian 2015). In the first subdomain, therefore, close to the surface (wall), a finer mesh distribution was used, whilst in the second (bulk region) a larger/ normal mesh distribution was constructed. Triangular mesh elements were used to establish the finite elements in each domain, Fig. 13. Mesh refinement was performed by analysing the mesh statistics changing from the COMSOL "extra fine" to the "extremely fine" mesh option. Increasing the number of triangular elements in this way did not show any significant change to the system behaviour. In summary, the mesh statistics showed that the minimum mesh element quality increased by $1.4 \%$, whilst the average element quality was increased by only $0.4 \%$. As was to be expected, for constant mesh area of $139.1 \mathrm{~mm}^{2}$, with a constant 12 vertex elements, the switch to the extremely fine mesh, raising the triangular element number from 34011 to 484013 , and the edge elements from 1533 to 2859 , resulted in a slowing of the maximum growth rate by $-4.9 \%$ and average growth rate by $-1.8 \%$. It was, therefore, decided to adopt the "extra fine" mesh system for the complete CFD analysis, as shown in Fig. 13.

A velocity profile applying nominal flow rate of $20 \mathrm{~m} \mathrm{~s}^{-1}$ is shown in Fig. 14. The formation of a stagnant layer (zero velocity) occurs both on the pixelated and flat surface, Fig. 14a, b, respectively, but importantly the pin-coated dots of active coating stand above the complete stagnation and penetrate the lower level of the continuous flow.

We can see in Fig. 15 that a significant aerofoil effect is induced as the flow across the pixelated (pin-coated) points occurs. It is this pressure drop, which can be as much $-25 \mathrm{~Pa}$, which is now investigated in respect to potential pressure-driven gas permeation through the active coating structure.

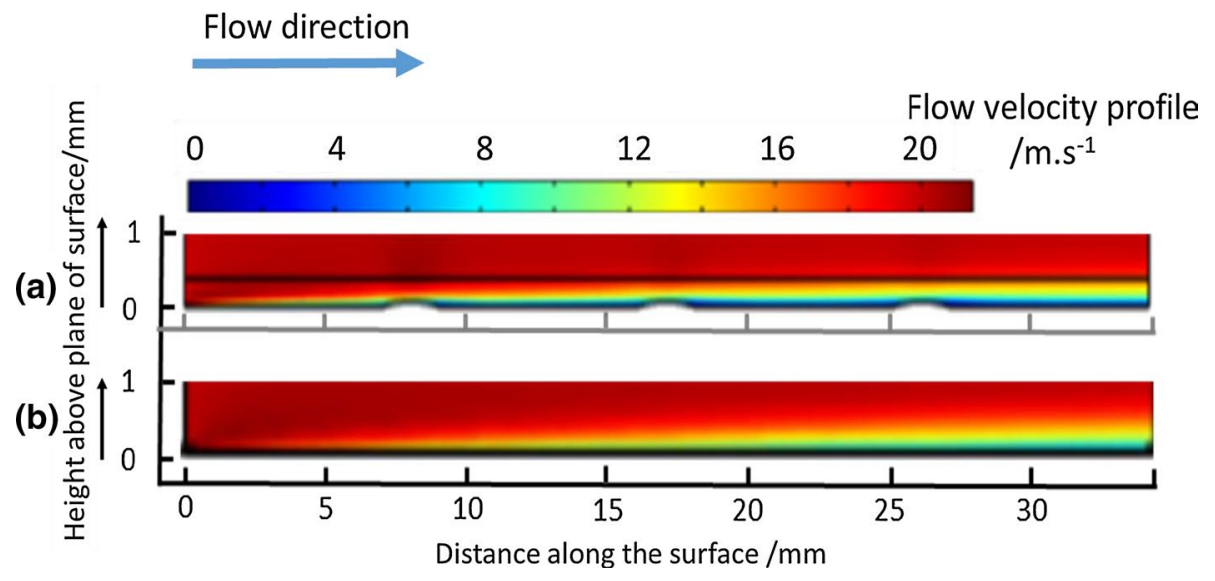

Fig. 14 Velocity profile at a nominal $20 \mathrm{~m} \mathrm{~s}^{-1}$ flow rate across a pixelated surface, and $\mathbf{b}$ flat surface 


\section{Flow direction}

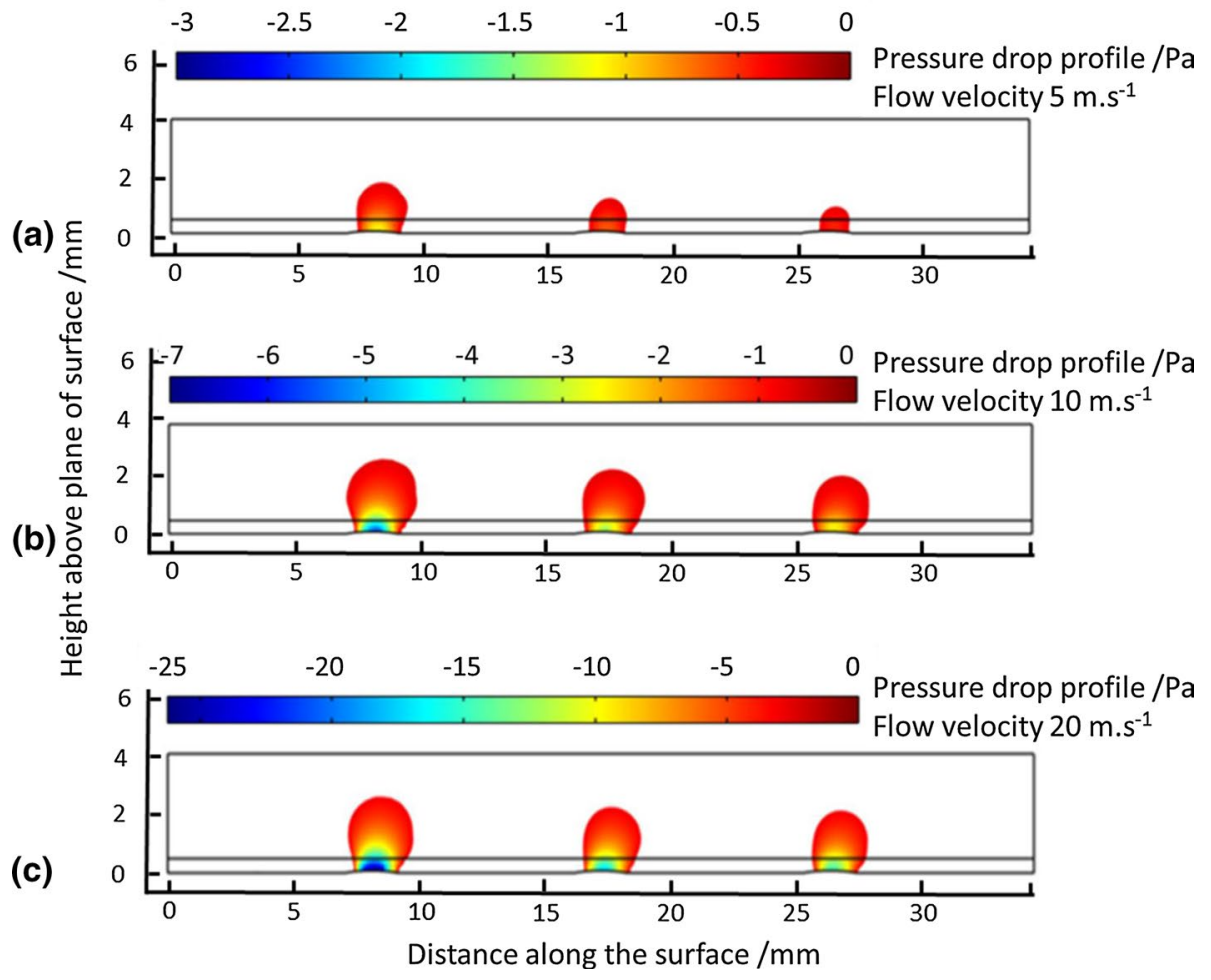

Fig. 15 Pressure drop contours across the pixelated surface at a 5, b 10 , and c $20 \mathrm{~m} \mathrm{~s}^{-1}$ nominal flow

\subsection{Pressure-Driven Permeation Versus Diffusion}

The permeation of $\mathrm{NO}_{2}$-in-air is shown schematically in Fig. 16, in which the direction of permeation is driven by the aerofoil pressure drop effect.

The pin-coated dot cross section is derived assuming a series of segments of a circle, radius $r$. The maximum permeation can be approximated by the maximum cross-section point, having area $A$, segment height $h$ and chord length $c$ (Fig. 15) and a path length of approximately $2 / 3$ of the dot diameter as follows,

$$
A=r^{2} \cos ^{-1}\{(r-h) / r\}-(r-h) \sqrt{ }\left(2 r h-h^{2}\right)
$$




\section{Bernoulli (aerofoil) effect, $\Delta P$}

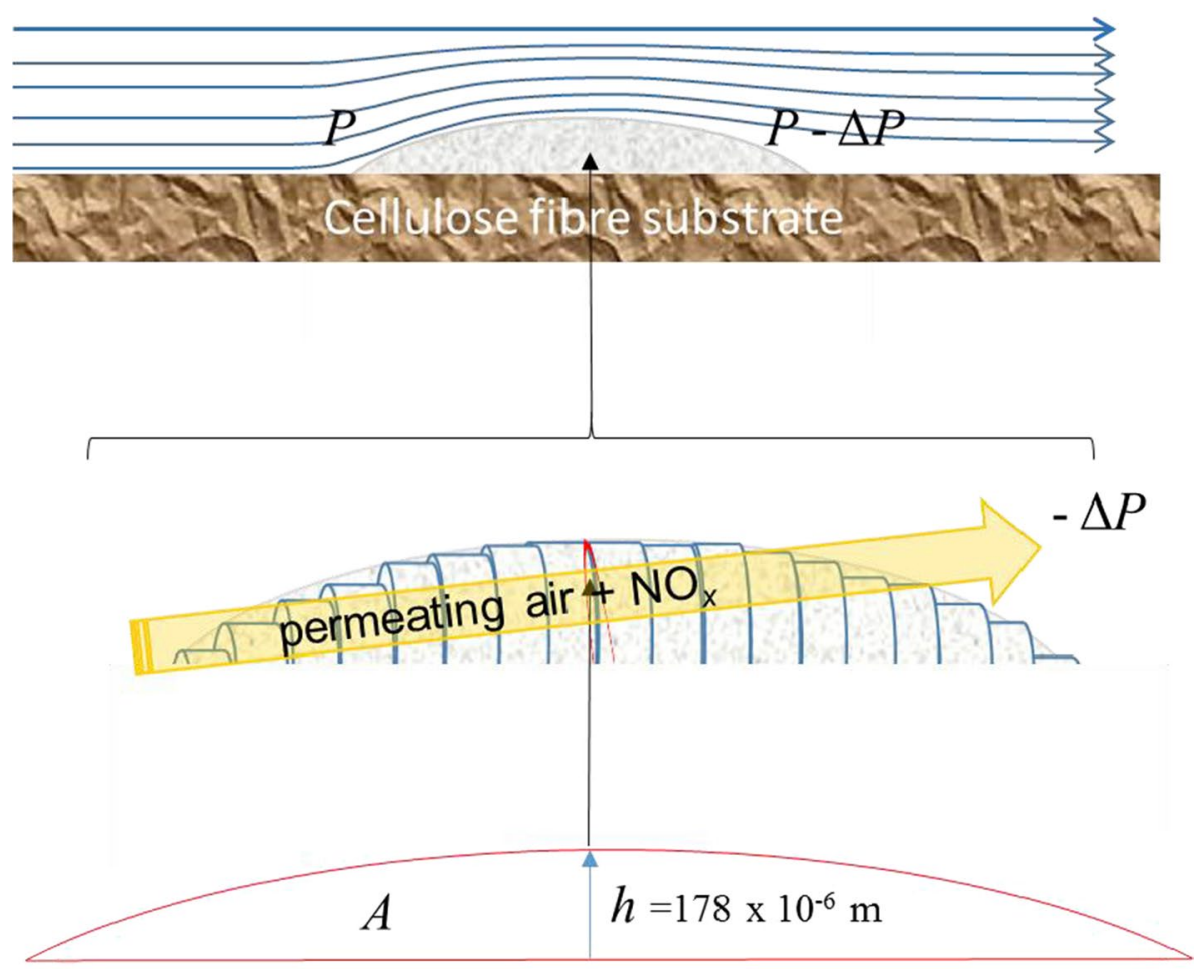

$$
c=2 \times 10^{-3} \mathrm{~m}
$$

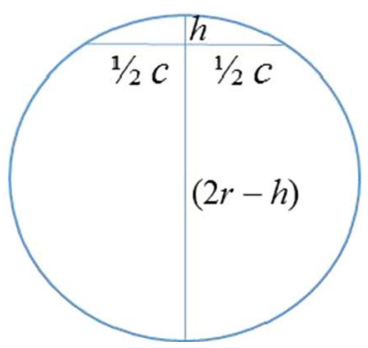

Fig. 16 Schematic of pressure-driven permeation caused by the Bernoulli aerofoil effect. The red outlined area represents the idealised cross-section sector area of the pin-coated dot

Since we do not know the radius of the circle a-priori, we can calculate it using the intersecting chords theorem, which states that the products of the intersected two lengths of each chord are equal. Thus using the geometry shown in the insert in Fig. 16, we have

$$
\begin{aligned}
& (1 / 2 c) \cdot(1 / 2 c)=h \cdot(2 r-h) \\
& \quad \Rightarrow 1 / 4 c^{2}=2 h r-h^{2} \\
& \quad \Rightarrow\left(1 / 4 c^{2}+h^{2}\right) / 2 h=r
\end{aligned}
$$




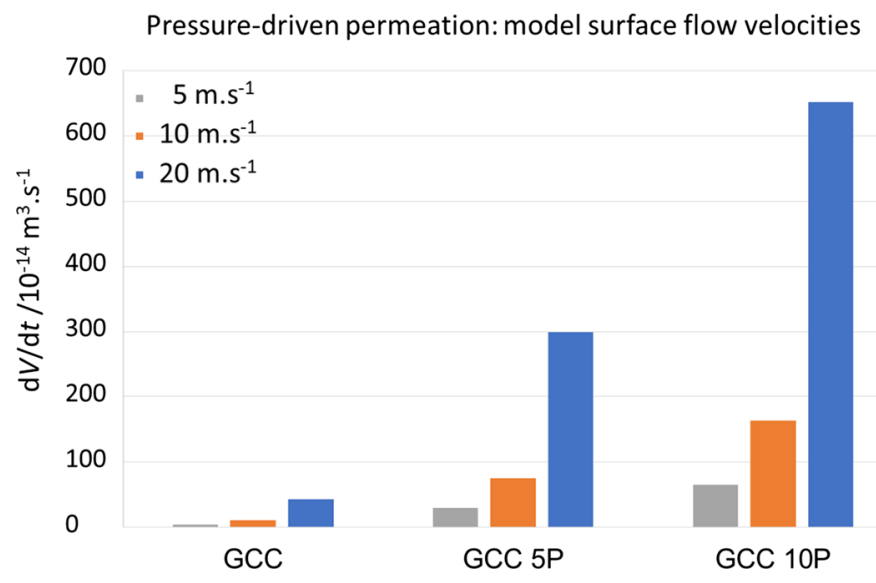

Fig. 17 Pressure-driven permeation flux for the range of surface-flow velocities and defined path length

Now putting $r$ into Eq. (9), we get

$$
\begin{aligned}
A= & \left.\left(\left(1 / 4 c^{2}+h^{2}\right) / 2 h\right)^{2} \cdot \cos ^{-1}\left\{\left(\left(1 / 4 c^{2}+h^{2}\right) / 2 h\right)-h\right) /\left(\left(1 / 4 c^{2}+h^{2}\right) / 2 h\right)\right\} \\
& -\left(\left(\left(1 / 4 c^{2}+h^{2}\right) / 2 h\right)-h\right) \sqrt{ }\left(1 / 4 c^{2}\right) \\
= & \left.\left(\left(1 / 4 c^{2}+h^{2}\right) / 2 h\right)^{2} \cdot \cos ^{-1}\left\{\left(\left(1 / 4 c^{2}+h^{2}\right) / 2 h\right)-h\right) /\left(\left(1 / 4 c^{2}+h^{2}\right) / 2 h\right)\right\} \\
& -\left(\left(\left(1 / 4 c^{2}+h^{2}\right) / 2 h\right)-h\right)(1 / 2 c)
\end{aligned}
$$

Applying the Darcy equation Eq. (2), we can insert the pressure drop values, $\Delta P$, to obtain the flux induced by the pressure effect. For the three different air velocities, 5,10 , and $20 \mathrm{~m} \mathrm{~s}^{-1}$, the values of $\Delta P$ are shown to be approximately 2,5 , and $20 \mathrm{~Pa}$, respectively. The permeation flow rates are shown for visual comparison in Fig. 17, for the parameters of gas properties, cross-sectional area, and path length, Table 9.

The comparison with diffusion can be followed by using Eqs. (4) and (8), the latter adopting the refined value for $\varepsilon_{\mathrm{t}}=p \phi$, where the cross-sectional area, $A_{\text {sample }}(=\pi \times($ $\left.2 \times 10^{-3} \mathrm{~m}\right)^{2} / 4=3.14 \times 10^{-6} \mathrm{~m}^{2}$ ), is now the area projection of the pin-coated dot and the path length is the dot height, $\sim 180 \mu \mathrm{m}$. Resulting values are given in Table 10. The values for $p \approx 0.4$ are a factor of 4 larger than quoted in the literature for coarse textured soils (Hunt 2004), indicating closer packing of finer, higher surface area particles than found in soil. A value of $p=0.4$ can therefore be used with some confidence for continuum diffusion modelling of fine particulate flocculated coatings.

The values for diffusion flux through such a short path length (the dot thickness only) versus that for the pressure-driven permeation, being $2 / 3$ of the dot diameter, are clearly 2-3 orders of magnitude greater. To make a more meaningful comparison, therefore, plots are shown in Fig. 18, which display the comparative flux between the two mechanisms, pressure-driven versus diffusion, in terms of the function of path length, $l$. Over the complete range of permeation lengths studied, the gas transport flux driven by diffusion is seen to be approximately 2 orders of magnitude greater than that driven by pressure. This finding is particularly relevant for the longer path lengths. However, for shorter distances, such as through the pin-coated point thickness the fast growth of the functions suggests that both are, in the limit of short path length, fast. The two effects, therefore, can be positively additive for very thin coatings. 


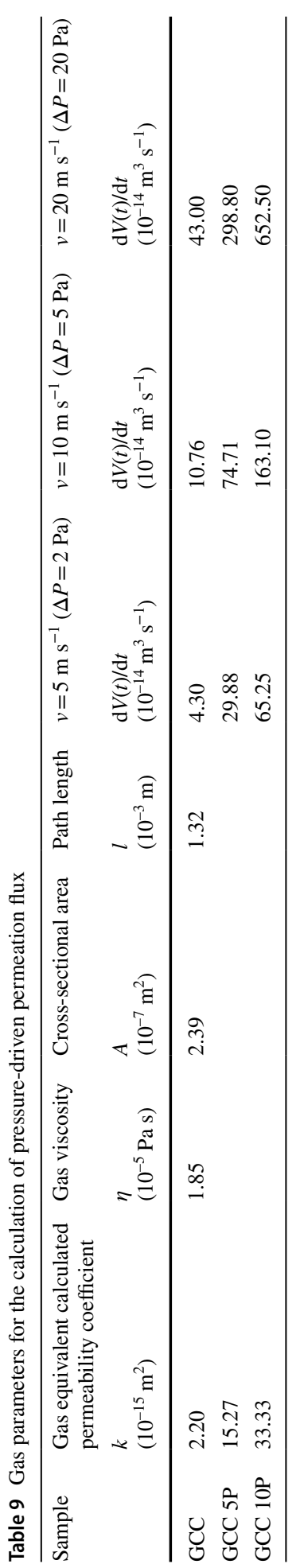




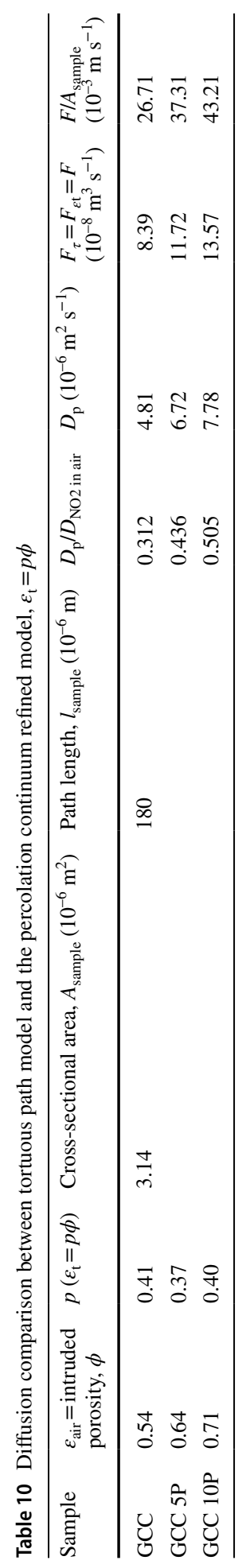


Fig. 18 Comparison of flux per unit cross-sectional area for a pressure-driven permeation versus $\mathbf{b}$ diffusion as a function of path length, $l$. The 2 orders of magnitude difference on the $y$ axes demonstrate the difference between the two mechanisms
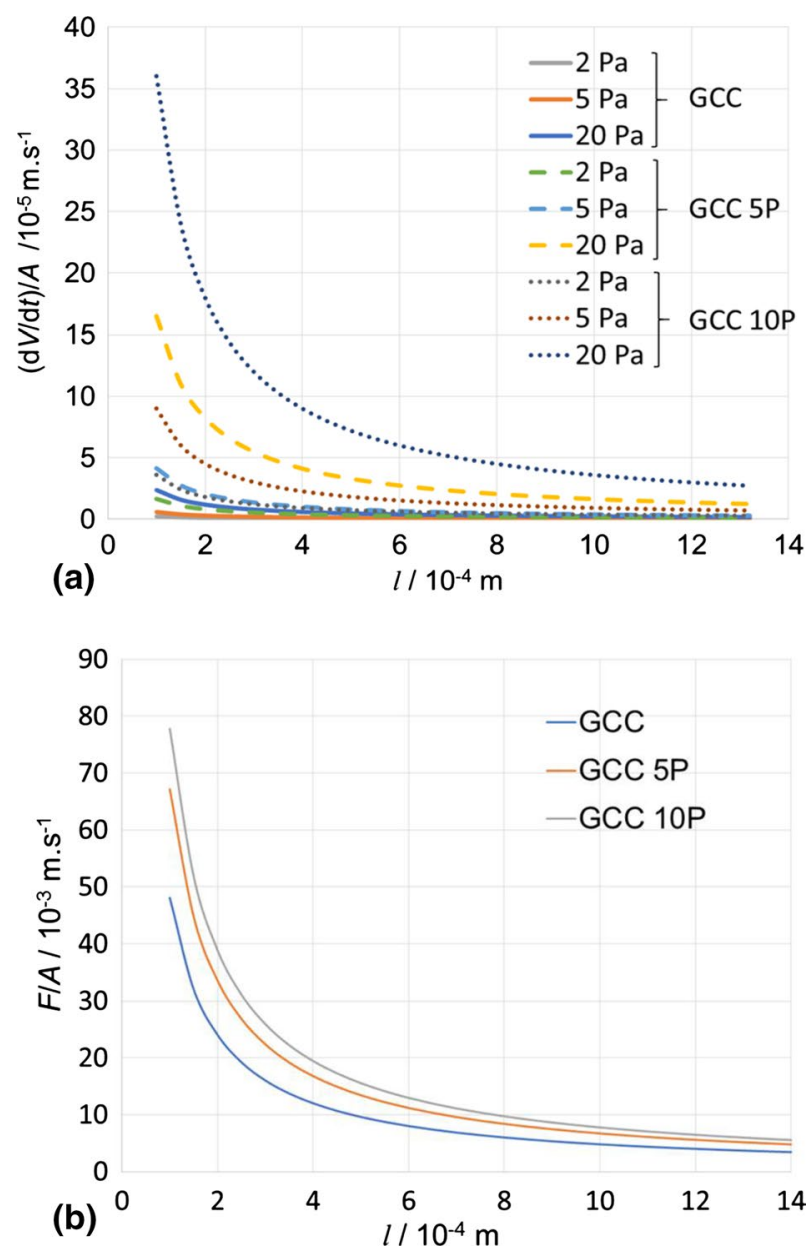

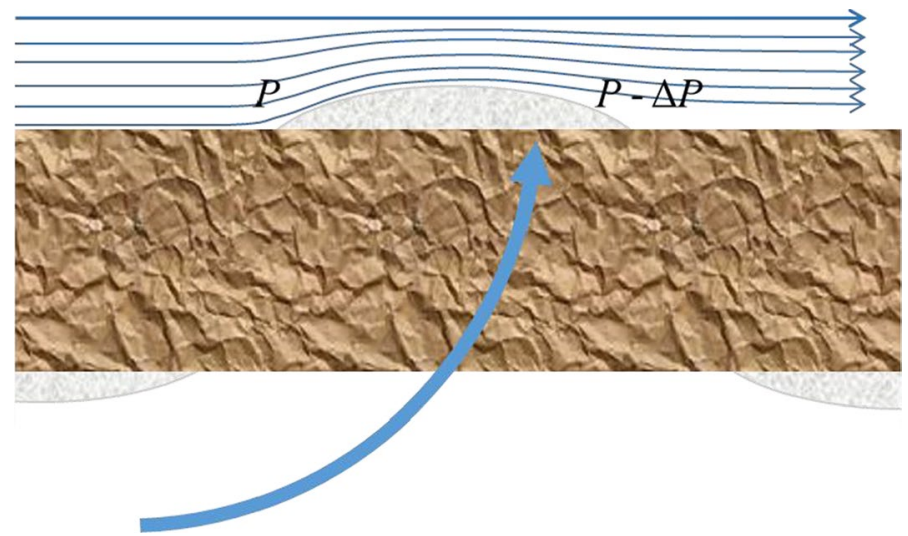

Fig. 19 Effect of pressure difference driving permeation of gas from the opposite side 
What's more, if we consider a permeability coefficient $\gtrsim 2$ orders of magnitude larger, typically expected for the fibrous substrate versus that of the mineral particle coating, then the pressure difference generated by the pixelated surface can readily drive permeation of gas from one side of the filter to the other in the case of the pin-coated sample, but this advantage is clearly lost for the flat surface and particularly for a highly impermeable continuous coating layer. Additionally, the pressure gradient between the sides, where on one side there is a pin-coated point and on the other uncoated substrate, leads to a vector director for the gas flow. This is illustrated schematically in Fig. 19.

\subsection{Transitioning from Bulk Flow Analysis to Pore-Scale Phenomena: Future Work}

The analysis presented in this paper has focused on the primary understanding of how to deliver the maximum gas volume transport within the porous medium of a coating in contact with a gas mixture under tangential lateral surface flow. The example used is the design of a reactive coating layer to capture $\mathrm{NO}_{x}$ via surface-flow filtration. As a result of the study, at this point, we are able to predict and quantify in relative terms the important bulk flow and gas separation criteria. However, the next stage of understanding the gasreactive coating would be to explore the diffusive and molecular dynamics behaviour on the individual pore scale. To achieve this, recent advances in 3D imaging offer the opportunity to capture the physical individual pore and pore cluster structure.

The increasingly many examples of the use of 3D imaging pertain frequently to spontaneous liquid imbibition in sandstone structures, related generally to wettability owing to its important role in the development of oil/gas reservoir dynamics. Prior art illustrates the employment of non-destructive X-ray tomography, for example in combination with lattice Boltzmann multiphase modelling (Lin et al. 2021). However, the spatial resolution in tomography is somewhat limited, reaching down to $0.07-0.1 \mu \mathrm{m}$ only after lengthy exposure of 24-30 h at high voltage $(\sim 8 \mathrm{kV})$ (Maire and Withers 2014), which can lead to material changes. Therefore, high-resolution semi-destructive techniques, such as sample sectioning in ultra-high resolution scanning electron microscopy (SEM) and focused ion beam scanning electron microscopy (FIB-SEM), are increasingly applied. Although the transform from a 2D image into a connected 3D pore structure is still a major challenge, image scaling provides the opportunity to consider the confines of a single pore, or even localised pore network. Visualising and quantifying the microstructure of oil shale prior to and after pyrolysis, using such higher resolution methods, was shown to be important in modelling hydrocarbon production potential. In this case, X-ray micro-tomography was combined with ultra-high resolution SEM and FIB-SEM (Saif et al. 2017). To improve imaging further, once again exemplified for the case of tight rocks, Lin et al. (2018) added extra information, available in terms of the fractal characteristics of the pores, to threshold segmentation based on the porosity model, and, in doing so, they attained the goal of increased resolution 3D imaging, reaching down to several nanometres.

Perhaps the most relevant reference to imaging pore network materials similar to those considered in this study, however, relates to focused ion beam ablation sectioning of specialised paper coating structures to illustrate the behaviour of liquid imbibition in inkjet printing (Aslannejad et al. 2017). Nonetheless, for gas, such an analysis would need to be extended to include compressibility. 


\section{Conclusions}

The study reported here has investigated the driving mechanisms for selective gas transport into a surface porous layer from a tangentially flowing gas mixture. The separation has been achieved via pore surface reactivity with the analyte gas $\mathrm{NO}_{2}$ to realise its capture from polluted air. Since the transport of gas is not driven by external applied pressure and size exclusion, but by concentration-driven diffusion and replenished by surface feature pressure differentiation, the mechanism differs strongly from existing tangential/cross-flow filtration. Thus, we use the term 'surface-flow' filter to describe the novel device. Wes how that in addition, by producing a pixelated patterned coating of active material on a highly porous substrate, the efficiency of surface-flow fluid sorption can be increased compared with a single continuous coating. The surface area in contact with the gas is increased per unit coat weight by a number of mechanisms. Firstly, for a flowing fluid, the stagnation layer can be penetrated by a surface feature even as little as a few hundred micrometres thick. Secondly, defining a pincoated printed dot, or similar, having surface curvature results in an aerofoil effect, which, though as seen here is insufficient to have a dramatic impact on lateral gas permeation, the surface replenishment of gas can be ensured, including the vertical (through-flow) pressuredriven permeation strongly supported by the pressure difference between the underside of the pin-coated dot and the surface exposed to flow acting across the large cross-sectional area of the dot in the $z$ direction $\left(\sim 3 \times 10^{-6} \mathrm{~m}^{2}\right)$ combined with the short path length of the dot thickness for the thin applied layer $(\leq 180 \mu \mathrm{m})$. In comparison, gas diffusion rate per unit cross-sectional area and given path length into the active coating structure is seen by modelling to be $\sim 2$ orders of magnitude greater. The combination of the two mechanisms results in an additive effect increasing the gas transport rate in the coating by $2 \%$. This effect, if designed correctly, can also result in gas contact with the underside of the active coating in addition to the top side due to pressure-driven flow through the much more porous fibrous substrate replenishing the gas reservoir for diffusion into the coating to take place.

One can conclude that the main benefit of pin coating is to provide diffusive access of the gas to the complete surface area of the coating dot, both above the substrate and in contact with it due to the extremely porous permeable cellulose fibre paper substrate. Thus, the surface area of the active coating accessible by the gas diffusing around and through the two side pin-coated filter is effectively double that of a continuous coating applied on two sides of the paper per unit coat weight. In contrast, a single continuous layer displays highly restrictive low permeability through the continuous coating layers, effectively reducing the gas accessibility to the exposed coating on the outside of the filter only, rendering the internal pores less efficient.

Acknowledgements The work was funded by Omya International AG, Switzerland, Group Sustainability. Thanks go to Silvan Fischer, microscopist at Omya International AG for the FESEM images of the compressed tabletted coating structures. The Nanotalo Microscopy Centre, Aalto University, provided the imaging facilities for the remaining electron micrographs.

Funding Open access funding provided by Aalto University. The research was funded by Omya International AG, Baslerstrasse 42, CH-4665 Oftringen, Switzerland.

Availability of data and materials All data are represented in the manuscript.

Code availability Software sourcing is indicated in the manuscript. 
Conflict of interest The authors declare that they have no known competing financial interests or personal relationships that could have appeared to influence the work reported in this paper.

Open Access This article is licensed under a Creative Commons Attribution 4.0 International License, which permits use, sharing, adaptation, distribution and reproduction in any medium or format, as long as you give appropriate credit to the original author(s) and the source, provide a link to the Creative Commons licence, and indicate if changes were made. The images or other third party material in this article are included in the article's Creative Commons licence, unless indicated otherwise in a credit line to the material. If material is not included in the article's Creative Commons licence and your intended use is not permitted by statutory regulation or exceeds the permitted use, you will need to obtain permission directly from the copyright holder. To view a copy of this licence, visit http://creativecommons.org/licenses/by/4.0/.

\section{References}

Aslannejad, H., Hassanizadeh, S.M., Raoof, A., de Winter, D.A.M., Tomozeiu, N., van Genuchten, M.T.: Characterizing the hydraulic properties of paper coating layer using FIB-SEM tomography and 3D pore-scale modeling. Chem. Eng. Sci. 160, 275-280 (2017). https://doi.org/10.1016/j.ces.2016.11.021

Barrett, E.P., Joyner, L.G., Halenda, P.P.: The determination of pore volume and area distributions in porous substances. I. Computations from nitrogen isotherms. J. Am. Chem. Soc. 73(1), 373-380 (1951). https://doi.org/10.1021/ja01145a126

Belfort, G., Davis, R.H., Zydney, A.L.: The behavior of suspensions and macromolecular solutions in crossflow microfiltration. J. Memb. Sci. 96, 1-58 (1994)

Berkowitz, B., Balberg, I.: Percolation theory and its application to groundwater hydrology. Water Resour. Res. 29, 775-794 (1993)

Brunauer, S., Emmett, P.H., Teller, E.: Adsorption of gases in multimolecular layers. J. Am. Chem. Soc. 60(2), 309-319 (1938). https://doi.org/10.1021/ja01269a023

Busatto, S., Vilanilam, G., Ticer, T., Lin, W.-L., Dickson, D.W., Shapiro, S., Bergese, P., Wolfram, J.: Tangential flow filtration for highly efficient concentration of extracellular vesicles from large volumes of fluid. Cells 7(12), 273 (2018). https://doi.org/10.3390/cells7120273

Cape, J.N.: Review of the use of passive diffusion tubes for measuring concentrations of nitrogen dioxide in air. RMP 2125 Variation Report, Edinburgh Research Station (2005)

Christy, C., Adams, G., Kuriyel, R., Bolton, G., Seilly, A.: High-performance tangential flow filtration: a highly selective membrane separation process. Desalination 144(1-3), 133-136 (2002)

Dehghani, M., Lucas, K., Flax, J., McGrath, J., Gaborski, T.: Tangential flow microfluidics for the capture and release of nanoparticles and extracellular vesicles on conventional and ultrathin membranes. Adv. Mater. Technol. 4(11), 1900539 (2019). https://doi.org/10.1002/admt.201900539

Dimić-Mišić, K., Gane, P.A.C., Paltakari, J.: Micro and nanofibrillated cellulose as a rheology modifier additive in CMC-containing pigment-coating formulations. Ind. Eng. Chem. Res. 52(45), 1606616083 (2013a). https://doi.org/10.1021/ie4028878

Dimić-Mišić, K., Puisto, A., Gane, P., Nieminen, K., Alava, M., Paltakari, J., Maloney, T.: The role of MFC/NFC swelling in the rheological behaviour and dewatering of high consistency furnishes. Cellulose 20, 2847-2861 (2013b). https://doi.org/10.1007/s10570-013-0076-3

Gane, P.A.C., Kettle, J.P., Matthews, G.P., Ridgway, C.J.: Void space structure of compressible polymer spheres and consolidated calcium carbonate paper-coating formulations. Ind. Eng. Chem. Res. J. 35(5), 1753-1764 (1996)

Gane, P.A.C., Dimić-Mišić, K., Barać, N., Imani, M., Janaćković, D., Uskoković, P., Barceló, E.: Unveiling a recycling-sourced mineral-biocellulose fibre composite for use in combustion-generated NOx mitigation forming plant nutrient: meeting sustainability development goals in the circular economy. Appl. Sci. 10, 3927 (2020). https://doi.org/10.3390/app10113927

Ghanbarian, B., Hunt, A.G.: Universal scaling of gas diffusion in porous media. Water Resour. Res. 50, 2242-2256 (2014)

Gribble, C.M., Matthews, G.P., Laudone, G.M., Turner, A., Ridgway, C.J., Schoelkopf, J., Gane, P.A.C.: Porometry, porosimetry, image analysis and void network modelling in the study of the pore level properties of filters. Chem. Eng. Sci. 66, 3701 (2011)

Hignett, T.P., ed.: Fertilizer Manual. Springer, Berlin (1985). ISBN 978-90-481-8290-9, ISBN 978-94017-1538-6 (eBook). https://doi.org/10.1007/978-94-017-1538-6

Hunt, A.G.: Applications of percolation theory to porous media with distributed local conductances. Adv. Water Resour. 24(3-4), 279-307 (2001) 
Hunt, A.G.: Continuum percolation theory for water retention and hydraulic conductivity of fractal soils: estimation of the critical volume fraction for percolation. Adv. Water Resour. 27, 175-183 (2004)

Hunt, A.G., Ewing, R.P.: On the vanishing of solute diffusion in porous media at a threshold moisture content. Soil Sci. Soc. Am. J. 67, 1701-1702 (2003)

Hunt, A.G., Ewing, R.P.: Percolation theory for flow in porous media. Lect. Notes Phys., 2nd ed., vol. 771. Springer, Berlin (2009)

Laudone, G.M., Matthews, G.P., Gane, P.A.C., Ridgway, C.J., Schoelkopf, J.: Estimation of the effective particle sizes within a paper coating layer using a void network model. Chem. Eng. Sci. 60, 6795 (2005)

Laudone, G.M., Matthews, G.P., Gane, P.A.C.: Modelling diffusion from simulated porous structures. Chem. Eng. Sci. 63, 1987 (2008)

Laudone, G.M., Gribble, C.M., Jones, K.L., Collier, H., Matthews, G.P.: Validated a priori calculation of tortuosity in porous materials including sandstone and limestone. Chem. Eng. Sci. 131, 109 (2015)

Launder, B.E., Sharma, B.I.: Application of the energy dissipation model of turbulence to the calculation of flow near a spinning disc. Lett. Heat Mass Transf. 1(2), 131-138 (1974)

Levy, C.L., Matthews, G.P., Laudone, G.M., Gribble, C.M., Turner, A., Ridgway, C.J., Gerard, D.E., Schoelkopf, J., Gane, P.A.C.: Diffusion and tortuosity in porous functionalized calcium carbonate. Ind. Eng. Chem. Res. 54(41), 9938-9947 (2015)

Li, J., Wang, Y., Wei, X., Wang, F., Han, D., Wang, Q., Kong, L.: Homogeneous isolation of nanocelluloses by controlling the shearing force and pressure in microenvironment. Carbohyd. Polym. 113, 388-393 (2014). https://doi.org/10.1016/j.carbpol.2014.06.085

Lin, W., Li, X., Yang, Z., Lin, L., Xiong, S., Wang, Z., Wang, X., Xiao, Q.: A new improved threshold segmentation method for scanning images of reservoir rocks considering pore fractal characteristics. Fractals 26(2), 1840003 (2018). https://doi.org/10.1142/S0218348X18400030

Lin, W., Xiong, S., Liu, Y., He, Y., Chu, S., Liu, S.: Spontaneous imbibition in tight porous media with different wettability: pore-scale simulation. Phys. Fluids 33, 032013 (2021). https://doi.org/10.1063/5. 0042606

Maire, E., Withers, P.J.: Quantitative X-ray tomography. Int. Mater. Rev. 59(1), 1-43 (2014). https://doi.org/ 10.1179/1743280413Y.0000000023

Menter, F.R.: Two-equation eddy-viscosity turbulence models for engineering applications. AIAA J. 32(8), 1598-1605 (1994). https://doi.org/10.2514/3.12149

Myan, F.W.Y., Min, L.K., Hong, L.C., Walker, J.: A numerical assessment of microtopographies with varied geometries in relation to biofouling control. J. Eng. Sci. Technol., 8th EURECA 2017 Special Issue August (2018), 67-78 [@ School of Engineering, Taylor’s University 67] (2018)

Palmes, E.D., Tomczyk, C.: Personal sampler for $\mathrm{NO}_{x}$. Am. Ind. Hyg. Assoc. J. 40, 588-591 (1979)

Price, J.C., Matthews, G.P., Quinlan, K., Sexton, J., Matthews, A.G.D.: A depth filtration model of straining within the void networks of stainless steel filters. AIChE J. 55(12), 3134-3144 (2009)

Ridgway, C.J., Schoelkopf, J., Gane, P.A.C.: A new method for measuring the liquid permeability of coated and uncoated papers and boards. Nord. Pulp Pap. Res. J. 18(4), 377-381 (2003)

Saif, T., Lin, Q., Butcher, A.R., Bijeljica, B., Blunt, M.J.: Multi-scale multi-dimensional microstructure imaging of oil shale pyrolysis using X-ray micro-tomography, automated ultra-high resolution SEM, MAPS Mineralogy and FIB-SEM. Appl. Energy 202, 628-647 (2017). https://doi.org/10.1016/j.apene rgy.2017.05.039

Schenker, M., Schoelkopf, J., Mangin, P., Gane, P.A.C.: Rheological investigation of complex micro nanofibrillated cellulose (MNFC) suspensions: discussion of flow curves and gel stability. Tappi J. 15(6), 405-416 (2016). https://doi.org/10.32964/TJ15.6.405

Schoelkopf, J., Ridgway, C.J., Gane, P.A.C., Matthews, P.G., Spielmann, D.C.: Measurement and network modelling of liquid permeation into compacted mineral blocks. J. Colloid Interface Sci. 227, 119-131 (2000). https://doi.org/10.1006/jcis.2000.6885

Sing, K.S.W.: Adsorption methods for the characterization of porous materials. Adv. Colloid Interface Sci. 76-77, 3-11 (1998). https://doi.org/10.1016/S0001-8686(98)00038-4

Stauffer, D., Aharony, A.: Introduction to percolation theory, 2nd edn. Taylor and Francis, London (1994)

Steward, D., Mayyas, A., Mann, M.: Economics and challenges of Li-ion battery recycling from end-of-life vehicles. In: 16th Global Conference on Sustainable Manufacturing-Sustainable Manufacturing for Global Circular Economy, Procedia Manufacturing, vol. 33, pp. 272-279 (2019)

Tabataian, M.: COMSOL ${ }^{\circledR} 5$ for engineers. Mercury Learning and Information, Herndon, VA, USA: ISBN 20172-0605ISBN 1942270429, 9781942270423, 312 p (2015)

van Reis, R., Gadam, S., Frautschy, L.N., Orlando, S., Goodrich, E.M., Saksena, S., Kuriyel, R., Simpson, C.M., Pearl, S., Zydney, A.L.: High performance tangential flow filtration. Biotechnol. Bioeng. 56(1), 
71-82 (1997). https://doi.org/10.1002/(SICI)1097-0290(19971005)56:1\%3c71::AID-BIT8\%3e3.0. $\mathrm{CO} ; 2-\mathrm{S}$

Vardoulakis, S., Fisher, B.E.A., Pericleous, K., Gonzalez-Flesca, N.: Modelling air quality in street canyons: a review. Atmos. Environ. 37, 155-182 (2003)

Vermesi, I., Restuccia, F., Walker-Ravena, C., Rein, G.: Carbon monoxide diffusion through porous walls: evidence found in incidents and experimental studies. Front. Built Environ. 4, 44 (2018). https://doi. org/10.3389/fbuil.2018.00044

Publisher's Note Springer Nature remains neutral with regard to jurisdictional claims in published maps and institutional affiliations. 